NBER WORKING PAPER SERIES

\title{
IMPLEMENTING OPTIMAL POLICY THROUGH INFLATION-FORECAST TARGETING
}

\author{
Lars E. O. Svensson \\ Michael Woodford \\ Working Paper 9747 \\ http://www.nber.org/papers/w9747

\section{NATIONAL BUREAU OF ECONOMIC RESEARCH 1050 Massachusetts Avenue Cambridge, MA 02138}

May 2003

Presented at NBER's Conference on Inflation Targeting, January 23-25, 2003, at Bal Harbour, Florida. We have benefited from discussions with and comments from the Giovanni Favara, Mark Gertler, Peter Ireland, Henrik Jensen, Mervyn King, Kai Leitemo, Bennett McCallum, Glenn Rudebusch, John Taylor, and participants in the conference and in seminars at Bank of Canada, Georgetown University, NBER's Monetary Economics Program, New York University, Princeton University, Université de Montréal and NBER's preconference. We also thank Giovanni Favara for research assistance and Christina Lönnblad and Kathleen DeGennaro for editorial and secretarial assistance. Remaining errors and expressed views are our own. The views expressed herein are those of the authors and not necessarily those of the National Bureau of Economic Research.

(C)2003 by Lars E. O. Svensson and Michael Wo. All rights reserved. Short sections of text not to exceed two paragraphs, may be quoted without explicit permission provided that full credit including (C) notice, is given to the source. 
Implementing Optimal Policy through Inflation-Forecast Targeting

Lars E. O. Svensson and Michael Woodford

NBER Working Paper No. 9747

May 2003

JEL No. E42, E52, E58

\section{$\underline{\text { ABSTRACT }}$}

We examine to what extent variants of inflation-forecast targeting can avoid stabilization bias, incorporate history-dependence, and achieve determinacy of equilibrium, so as to reproduce a socially optimal equilibrium. We also evaluate these variants in terms of the transparency of the connection with the ultimate policy goals and the robustness to model perturbations. A suitably designed inflation-forecast targeting rule can achieve the social optimum and at the same time have a more transparent connection to policy goals and be more robust than competing instrument rules.

Lars E. O. Svensson

Department of Economics

Fisher Hall

Princeton University

Princeton, NJ 08544-1021

and NBER

svensson@princeton.edu

\author{
Michael Woodford \\ Department of Economics \\ Fisher Hall \\ Princeton University \\ Princeton, NJ 08544-1021 \\ and NBER \\ woodford@princeton.edu
}




\section{Contents}

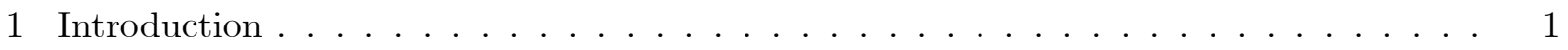

1.1 Disadvantages of purely forward-looking policymaking . . . . . . . . . . . 1

1.2 Monetary-policy rules and approaches to policy implementation . . . . . . . . 5

2 The model . . . . . . . . . . . . . . . . . . . . . . . 9

2.1 Optimal equilibrium responses to shocks . . . . . . . . . . . . . 15

2.2 Optimality from a "timeless perspective" . . . . . . . . . . . . . . . 18

2.3 Interest rates in an optimal equilibrium . . . . . . . . . . . . . . 22

2.4 The problem of indeterminacy . . . . . . . . . . . . . . . . . 26

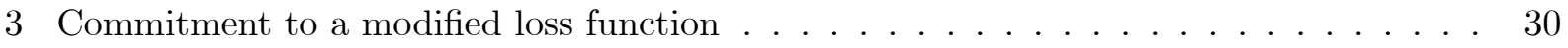

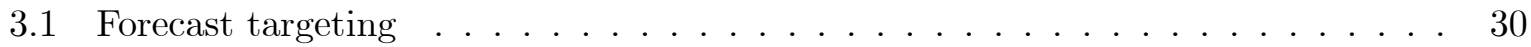

3.2 Discretionary minimization of the social loss function . . . . . . . . . . . . 31

3.3 A dynamic-programming procedure . . . . . . . . . . . . . . . . 33

3.4 Sequentially constrained optimization . . . . . . . . . . . . . . 35

3.5 Minimization of a modified loss function: "Commitment to continuity and pre-

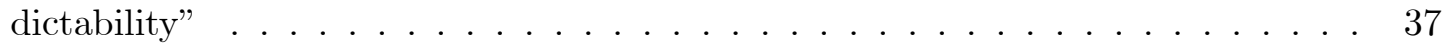

3.5.1 An explicit decision procedure . . . . . . . . . . . . . 38

3.5.2 The implied reaction function and determinacy . . . . . . . . . . . 43

3.6 A hybrid rule that ensures determinacy . . . . . . . . . . . . . . . . . . 44

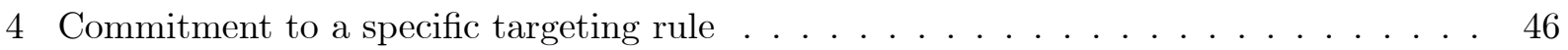

4.1 Determinacy under the specific targeting rule . . . . . . . . . . . . . 48

4.2 A hybrid rule related to the specific targeting rule $\ldots \ldots \ldots \ldots$

4.3 A commitment to a an equivalent specific price-level targeting rule . . . . . . . 51

5 Commitment to an explicit instrument rule . . . . . . . . . . . . . . 54

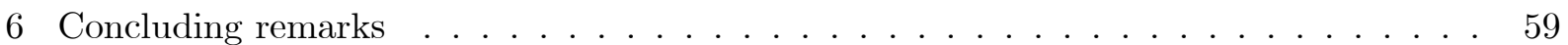

A The necessary and sufficient conditions for determinacy $\ldots \ldots \ldots \ldots$ 


\section{Introduction}

In recent years, many central banks have adopted "inflation targeting" frameworks for the conduct of monetary policy. These have proven in a number of countries to be effective means of first lowering inflation and then maintaining both low and stable inflation and inflation expectations, without negative consequences for the output gap. Thus, the new approach to monetary policy has been judged quite successful, as far as its consequences for the average level of inflation and the output gap are concerned.

It has been less clear how effective these procedures are as ways of bringing about desirable transitory fluctuations in inflation and output in response to exogenous shocks. ${ }^{1}$ But this is also a relevant question in the choice of a framework for the conduct of monetary policy; moreover, the expectation that inflation targeting procedures will perform well in this respect is often cited as one of their leading advantages over other approaches to the maintenance of low inflation and the achievement of credibility. For example, King [16] argues the superiority of inflation targeting over commitment to a money-growth rule on the ground that, while either approach should equally serve to maintain low average inflation and low inflation expectations, inflation targeting also results in optimal short-run responses to shocks, while money-growth targeting does not. Here we consider how inflation targeting should be conducted in order to achieve this goal.

\subsection{Disadvantages of purely forward-looking policymaking}

In King's analysis, "inflation targeting" is associated with decision-making under discretion. However, that discretion is constrained by a clear objective, involving inflation stabilization around the inflation target and output-gap stabilization around an output-gap target. In particular, the output-gap target is modified (relative to the output gap target that would reflect true social preferences) to equal zero, so as to be consistent with the natural output level. This modification of the output-gap target suffices to eliminate the "average inflation bias" associated with discretionary policymaking, and in the simple Barro-Gordon model that King assumes, this also suffices to make the outcome of discretionary optimization fully optimal, that is, consistent with the optimal equilibrium under commitment, including optimal responses to transitory shocks.

However, this result is quite special to the simple model that King uses. As a number of

\footnotetext{
${ }^{1}$ See, for instance, Svensson [36], especially footnote 43.
} 
authors have pointed out, in the presence of forward-looking private-sector behavior (of the kind that naturally results from dynamic optimization by the private sector), discretionary optimization by a central bank generally results not only in average inflation bias, when the output-gap target is positive, but also in inefficient responses to shocks (what is sometimes called "stabilization bias"), regardless of whether the output-gap target is positive or not. ${ }^{2}$

The reason is simple. In general, forward-looking behavior implies that the bank's short-run tradeoffs (between, say, its inflation stabilization and output-gap stabilization) following a shock can be improved if it can be arranged for private-sector expectations about future inflation and output to adjust in the right way in response to the shock. However, this can occur-when the private sector has rational expectations - only if subsequent central bank policy does in fact change as a result of the past shocks, in such a way as to bring about the alternative evolution that it was desired that people would expect. But under discretionary optimization, it will not, as the central bank will re-optimize afresh at the later date, and care nothing about past conditions that no longer constrain what it is possible for it to achieve at that date. This problem can exist, and generally does, even when the output-gap target is consistent with steady inflation at the inflation target so that there is no average inflation bias.

As Woodford [45] stresses, the suboptimal responses to shocks characteristic of discretionary optimization also characterize any decision procedure for monetary policy that is purely forwardlooking. By a purely forward-looking procedure we mean one in which only factors that matter for the central bank's forecast of the future evolution of its target variables, conditional upon its current and future policy actions, play any role in its decisions. Any such procedure has the property that, if it determines a unique equilibrium, that equilibrium is one in which the evolution of the target variables depends only upon the factors just mentioned. In particular, the equilibrium paths of the target variables will be independent of past conditions that no longer matter for current equilibrium determination except insofar as the central bank may condition its policy upon them. But, as Woodford [44] emphasizes, in general forward-looking private-sector behavior implies that an optimal equilibrium will involve additional history-dependence. This is because it is optimal for the path of the target variables to depend upon past conditions - even

\footnotetext{
${ }^{2}$ Jonsson [12] and Svensson [34] point out that stabilization bias and conditional inflation bias, as distinct from average inflation bias, arises in a Barro-Gordon model with output persistence, that is, with an endogenous state variable. Flodén [9], Clarida, Galí and Gertler [4] and Woodford [44] show that stabilization bias arises with a Calvo-type forward-looking Phillips curve. The problem goes beyond a mere contemporaneous response to shocks of the wrong size. Instead, as stressed by Woodford [44] and [45], discretionary optimization also generally leads to a suboptimal degree of persistence of the effects of shocks as well - the problem of inadequate history-dependence discussed below.
} 
when these no longer constrain currently feasible outcomes - because of the effects of the prior anticipation of such dependence upon the path of the target variables at earlier dates. ${ }^{3}$

Purely forward-looking approaches to monetary policy are also more easily prone to another problem, which is indeterminacy of rational-expectations equilibrium. Most inflation-targeting central banks (as, indeed, most central banks nowadays) use a short-term nominal interest rate as the policy instrument or "operating target". But as Sargent and Wallace [30] first stressed, interest-rate rules may allow a large multiplicity of rational-expectations equilibrium paths for real and nominal variables, including equilibria in which fluctuations occur that are unrelated to any variation in economic "fundamentals". This indeterminacy is plainly undesirable - at least if alternative policy rules are available, that are equally consistent with the best equilibrium, but do not allow the bad ones - since some of the possible equilibria will be very bad, from the point of view of any objective that penalizes unnecessary variation in the target variables. ${ }^{4}$

In the case of many forward-looking models derived from private-sector optimization, as with the rational-expectations IS-LM model analyzed by Sargent and Wallace [30], one can show that commitment to any reaction function that determines the path of the nominal interest rate purely as a function of exogenous factors (that is, without any feedback from endogenous variables such as the rate of inflation) implies indeterminacy of the equilibrium price level. ${ }^{5}$ However, this does not mean that interest-rate-setting procedures as such must lead to this outcome; as McCallum [22] first noted, a sufficient degree of dependence (of the right sort) of the central bank's interest-rate operating target upon endogenous variables can render equilibrium determinate, in the sense of there existing a unique non-explosive solution to the equilibrium conditions. It is important, though, to choose an interest-rate-setting procedure that involves sufficient dependence of this kind.

One example of the kind of dependence that suffices for determinacy in the simple forwardlooking model used below is that assumed in the well-known reaction function proposed by Taylor [40]: making the nominal interest rate an increasing function of the observed inflation and output gap, with a positive coefficient on the output gap and a coefficient greater than one on inflation. This sort of reaction function has also been found to lead to a determinate

\footnotetext{
3 The history-dependence of equilibria resulting from optimal policy under commitment in the case of a forward-looking system has been observed since the early treatments by, for instance, Backus and Driffill [1] and Currie and Levine [7].

4 This criterion for choice among alternative monetary-policy reaction functions is also stressed in Bernanke and Woodford [2], Christiano and Gust [5], Clarida, Galí and Gertler [3], Kerr and King [14], Rotemberg and Woodford [28], and Woodford [44] and [47, chapter 4].

${ }^{5}$ See Woodford [44] for a result of this kind in the context of a model closely related to that used here.
} 
equilibrium in a variety of other types of forward-looking models. ${ }^{6}$

The kind of dependence that is needed for determinacy may not be possible in the case of a purely forward-looking procedure of the kind often assumed in discussions of inflationforecast targeting. To make this point in an especially sharp way, we here consider a simple forward-looking model in which no lagged endogenous variables matter for the determination of future inflation and output. In this case, a purely forward-looking monetary-policy procedureby which (in line with Woodford [46] and Giannoni and Woodford [11]) we mean one under which the decision at each point in time depends only on the set of possible future paths for the economy, given its current condition - must make the central bank's instrument choice a function solely of information about the future evolution of the exogenous disturbances. Under the further assumptions that (i) all information about the exogenous disturbances that is available to the private sector is also directly observed by the central bank, and (ii) the central bank must choose its current instrument setting before observing the private sector's current choices of endogenous variables and its current expectations, this means that the nominal interest rate will evolve solely as a function of exogenous state variables, independent of the paths of any of the endogenous variables. But such a rule implies indeterminacy of the equilibrium paths of both inflation and output. $^{7}$

Thus, we conclude once again that a decision procedure that can be relied upon to achieve the optimal equilibrium under commitment must be history-dependent in a way that purely discretionary decision-making procedures are not, as well as insure determinacy of the equilibrium. Our task in this paper is to consider to what extent various alternative forms of inflation targeting can avoid stabilization bias, incorporate history-dependence of the proper sort and result in determinacy of the equilibrium.

\footnotetext{
${ }^{6}$ See Christiano and Gust [5], Levin, Wieland and Williams [21], Rotemberg and Woodford [28], and Woodford $[44]$.

7 Studies such as Clarida, Galí and Gertler [3], and Woodford [47, chapter 4], find that equilibrium may be determinate, in a forward-looking model closely related to our own, under commitment to a rule that makes the nominal interest rate a sufficiently sharply increasing function of current and/or expected future inflation and output gaps over some horizon. But their result is obtained by assuming that the desired relation between expected inflation and output and the nominal interest rate can be imposed as an equilibrium condition: the bank's ability to ensure that it necessarily holds in equilibrium is not questioned. Such a condition, however, is an implicit instrument rule and does not represent a fully operational specification of the monetary policy rule, as the central bank's instrument is expressed as a function of endogenous variables (conditional expectations of future inflation and output) that themselves depend upon current monetary policy. In practice, the bank would have to forecast the paths of the endogenous variables, given its contemplated action. If this forecast depends only on information about the exogenous disturbances and the bank's contemplated policy, then an operational version of the policy rule, an explicit instrument rule, in which the bank's decision procedure is completely specified as an algorithm, is equivalent to a rule that sets the nominal interest rate as a function of the exogenous disturbances, and leads to indeterminacy.
} 


\subsection{Monetary-policy rules and approaches to policy implementation}

Since we will discuss the details of alternative decision frameworks for monetary policy, it is practical to have a consistent classification of such decision frameworks. In this paper, as in Svensson [36] and [38], a "monetary-policy rule" is interpreted broadly as a "prescribed guide for monetary-policy conduct." We give particular attention to a special type of policy rules, that we call "targeting rules." "Target variables" are endogenous variables that enter a loss function, a function that is increasing in the deviations of the target variables from prescribed "target levels." "Targeting" is minimizing such a loss function. "Forecast targeting" refers to using forecasts of the target variables effectively as intermediate target variables, as in King's [15] early characterization of inflation targeting.

A "general targeting rule" is a high-level specification of a monetary-policy rule that specifies the target variables, the target levels and the loss function to be minimized. A complete description of such a procedure also requires specification of the exact procedure used to determine the actions that should minimize the loss function, such as the one that we propose in section 3 below.

A "specific targeting rule" is instead expressed directly as a condition for the target variables, a "target criterion." Under certain circumstances, commitment to a general targeting rule may be equivalent to a particular specific targeting rule, which describes conditions that the forecast paths must satisfy in order to minimize a particular loss function. Nonetheless, it may be important to distinguish between the two ways of describing the policy commitment, on grounds either of differing efficiency as means of communicating with the public, or of differing degrees of robustness to changes in the model of the economy used to implement them. Furthermore, a specific targeting rule need not be equivalent to any intuitive general targeting rule, ${ }^{8}$ and indeed one of our primary reasons for interest in such specifications here will be their greater flexibility, making it easier to introduce history-dependence of the sort required to solve the problems introduced in the previous section.

Any policy rule implies a "reaction function," that specifies the central bank's instrument as a function of predetermined endogenous or exogenous variables observable to the central bank at the time that it sets the instrument. This "implied reaction function" should not, in general, be confused with the policy rule itself; for example, the implied reaction function associated with a

\footnotetext{
${ }^{8}$ One can always find a trivial general targeting rule for any specific targeting rule by simply letting the loss function be the square of the specific targeting rule written as a target criterion equal to zero.
} 
given policy rule will generally change in the case of changes in the model of the economy used in implementing the rule. However, an "explicit instrument rule" is a low-level specification of the monetary-policy rule, in the form of a prescribed reaction function. Proposals such as the policy rule advocated by Taylor [40] are of this form.

We are interested in decision procedures for monetary policy that can achieve (or at least come close to) the optimal equilibrium under commitment. In fact, there is no single policy rule that is uniquely consistent with the optimal equilibrium. Many rules may be consistent with the same equilibrium, even though they are not equivalent insofar as they imply a commitment to different sorts of out-of-equilibrium behavior. Furthermore, even rules that specify the same actions in all circumstances, given a particular model of the economy, may deserve separate consideration because they would no longer be equivalent if the bank's model of the economy were to change.

We shall not here attempt to enumerate all of the possible types of policy rules that could achieve the optimal equilibrium. Instead, we shall seek approaches to this problem that preserve, to the greatest extent possible, the attractive features of "inflation-forecast targeting," the procedure currently used (in one variant or another) by the most prominent inflation-targeting central banks. ${ }^{9}$ For example, we shall prefer approaches in which the decision process has as transparent a connection as possible with the central bank's ultimate objectives. A procedure like inflation-forecast targeting, in which the entire decision process is organized around the pursuit of an explicit objective defined in terms of the ultimate goal variables, has several advantages. Focus upon such an objective helps to ensure that policy is made in a coherent fashion; it facilitates communication with the public about the intended consequences of the bank's policy, even when the full details of the implementation of the policy may be too complex to describe; and it favors accountability by indicating the way in which the policy's success can appropriately be measured. We shall inquire as to the extent to which we can preserve this sort of transparency while introducing the sort of history-dependence required for a determinate equilibrium with optimal responses to shocks.

Another criterion for a good policy rule is robustness of the rule specification to possible changes in the details of the bank's model of the economy. A full analysis of the question of robustness would necessarily be numerical, as in general one cannot expect any rule to be completely unaffected by possible model changes, and the question will be which kinds of rules

\footnotetext{
${ }^{9}$ See, for instance, Svensson [33], [36] and [38] for discussion of procedures of this general type.
} 
are less affected. Nonetheless, we here consider robustness of a somewhat special kind, which is the possibility that a rule may continue to be optimal under some particular (restricted) class of perturbations of the model. On this ground, we shall consider a policy rule better if it continues to be optimal under a larger class of perturbations than is true for another rule.

This, too, is a desirable feature of inflation-forecast targeting proposals. These tend to be high-level specifications of monetary policy, with the details of implementation depending upon the details of the particular model of the economy used by a particular central bank. In some cases, changes in the model require no change in the high-level description of optimal policy. For example, Svensson [33] and [38] show how a targeting rule defined in terms of desired features of the forecast paths for inflation and the output gap may correspond to a first-order condition that characterizes the optimal equilibrium. An advantage of this way of describing the optimal equilibrium is that the form of the first-order condition is invariant under certain changes in the model, notably changes in the assumed character of (additive) stochastic disturbances. Here we shall give attention to policy specifications that share this property, though they involve history-dependence sufficient to eliminate the problems just mentioned with purely forwardlooking procedures. ${ }^{10}$

With these desiderata in mind, we explore the possibility of implementing the optimal equilibrium in each of three possible ways. Our highest-level policy specification is in terms of a general targeting rule, a loss function that the central bank is committed to seeking to minimize, through a forecast-based dynamic optimization procedure. In the case of this way of specifying policy, the history-dependence necessary for optimality must be introduced through a modification of the central bank's loss function, that must be made history-dependent in a way that the true (social) loss function is not.

Our second, intermediate-level policy specification is in terms of a specific targeting rule, specifying a criterion that the bank's forecast paths for its target variables must satisfy. This kind of rule specifies a relation involving one or more endogenous variables that cannot be directly observed at the time that policy is chosen, and that instead must be forecasted. Furthermore, in the case of a forward-looking model, even forecasting endogenous variables a short time in the future will in general require solving for the model's equilibrium into the indefinite future; thus a forecast of the entire future paths of the various variables is required. A decision procedure of this kind is therefore still organized around the construction of forecast paths conditional

\footnotetext{
10 In Svensson [33], problems of stabilization bias and lack of history-dependence do not arise, owing to the absence of forward-looking elements in the simple model used to expound the idea.
} 
upon alternative policies, even if explicit optimization is not undertaken. In the case of such a targeting rule, the history-dependence necessary for determinacy and optimality must be introduced through commitment to a rule that involves lagged endogenous variables as well as forecasts of their future values.

Finally, our lowest-level specification of policy is in terms of an explicit instrument rule, specifying the setting of the central bank's instrument as a function of variables that are exogenous or predetermined at the time. Implementation of this kind of policy rule is no longer dependent upon either a model of the economy or an explicit objective function. We find that such rules are less transparently related to the ultimate objectives of policy than in the other two cases, also when we consider the possibility of instrument rules that are relatively robust to changes in model specification, owing to their derivation from first-order conditions that characterize the optimal equilibrium. Such rules also differ from the other two cases in that they are purely backward-looking; as a result, introduction of the dependence upon lagged endogenous variables required for determinacy and optimality is straightforward.

Our analysis leads us to more than one example of a policy rule that both renders equilibrium determinate and achieves the optimal equilibrium, if the central bank's commitment to it can be made credible to the private sector. These include history-dependent variants of inflationforecast targeting. We thus conclude that the need for history-dependence in policy, for the reasons just sketched, is consistent with a suitably designed forecast-targeting procedure.

The paper is organized as follows. In section 2, we introduce a simple forward-looking model that allows us to make the above remarks more concrete. We characterize the optimal equilibrium in such a model, and show that it involves history-dependence of a kind not consistent with purely discretionary decisionmaking. We also show that the problem of indeterminacy of equilibrium arises in this model and needs to be considered in the specification of the different policy rules.

In sections 3, 4 and 5, we then take up the three successively lower-level specifications of policy described above. In each case, we consider ways in which the sort of history-dependence in policy required for consistency with the optimal equilibrium can be introduced. We also treat the issue of determinacy of equilibrium for each of the policies analyzed. Finally, in section 6 , we compare the advantages and disadvantages of the various proposals taken up in the previous sections. Here we also briefly discuss the transparency of the connection to policy goals and the robustness of our various policy specifications. We conclude that a variant of inflation-forecast 
targeting, modified to include a commitment by the central bank to respond to deviations of private-sector expectations from those it had forecasted, represents an especially attractive procedure from the point of view of these several criteria.

\section{The model}

The model is a variant of a standard forward-looking model used, for example, in Clarida, Galí and Gertler [4] and Woodford [44] and [47]. In the variant that we use here, inflation and output are both predetermined for one period, as in Bernanke and Woodford [2], Rotemberg and Woodford [27] and [28], and Svensson [38], except for an unforecastable random error term that cannot be affected by monetary policy. Optimizing private-sector behavior is represented by two structural equations, an aggregate-supply equation (derived from a first-order condition for optimal price-setting by the representative supplier) and an "expectational IS curve" (derived from an Euler equation for the optimal timing of purchases). ${ }^{11}$

The forward-looking aggregate-supply (AS) equation takes the form

$$
\pi_{t+1}=\beta \pi_{t+2 \mid t}+\kappa x_{t+1 \mid t}+u_{t+1},
$$

where $\pi_{t+1}$ is inflation between periods $t$ and $t+1$ (also referred to as inflation in period $t+1$ ), $x_{t}$ is the output gap, indicating the percentage by which output exceeds potential, $0<\beta<1$ is a discount factor, $\kappa$ is a positive coefficient, and $u_{t+1}$ is an exogenous disturbance term, the value of which is realized only in period $t+1 .{ }^{12}$ For any variable $z$ and any horizon $\tau \geq 0$, we use the notation $z_{t+\tau \mid t} \equiv \mathrm{E}_{t} z_{t+\tau}$ to denote private-sector expectations regarding $z_{t+\tau}$ conditional on information available in period $t$; for example, $\pi_{t+2 \mid t}$ denotes private-sector inflation expectations in period $t$ of inflation between periods $t+1$ and $t+2$. This variant of the Calvo-Rotemberg aggregate-supply relation differs from that used, for example, in Woodford [44] in that the conditional expectations of $x_{t+1}$ and $\pi_{t+2}$ are taken in period $t$ rather than $t+1$. This is because, except for the surprise component $u_{t+1}-u_{t+1 \mid t}$, we assume that prices are determined one period in advance. As a result of this decision lag, the first-order condition for "voluntary"

\footnotetext{
${ }^{11}$ See Woodford [47] for general discussion of the microeconomic foundations of the class of models to which ours belongs.

${ }^{12}$ Here we assume, as in standard expositions of the Calvo pricing model, that prices remain fixed in monetary terms between the occasions on which they are re-optimized. It is worth noting, however, that if we were to assume a constant rate of increase in prices between the occasions on which prices are re-optimized, as in Yun [48], the aggregate-supply relation would take the same form, but with $\pi_{t+1}$ interpreted as inflation in excess of that "normal" rate. Our conclusions below as to the character of optimal policy would also all have direct analogs in that case, allowing for the possibility of optimal targeting rules in which the inflation target could differ from zero.
} 
price changes is the same as in the simpler case, but conditioned upon an earlier information set. This has the consequence that, as is often assumed, monetary policy changes will have no effect upon inflation within the period in which the change first becomes public. We assume that measured inflation differs from the average of "voluntary" price changes by an error term that need not be forecastable when the "voluntary" price changes are determined; this might be interpreted either as measurement error in the price index, or as a time-varying markup of retail prices over the predetermined wholesale prices. ${ }^{13}$ We allow for the existence of a "surprise" component of inflation in order to avoid the counterfactual implication that inflation is known with perfect certainty one period in advance.

Our specification also differs from the simplest one in that we allow for a forecastable "costpush" shock $u_{t+1 \mid t}$, which shifts the distance between "potential output" (with respect to which our "output gap" is defined) and the level of output that would be consistent with zero "voluntary" inflation. Thus, we assume that some exogenous shifts in the aggregate supply curve do not correspond to changes in the efficient level of output (an example would be exogenous variation in the markup over wholesale prices); these shifts are not considered to represent variation in "potential output" (so that the social loss function can still be expressed in terms of our output-gap variable), and thus appear as a residual in (2.1). Allowance for such a shock creates a conflict between inflation stabilization and output-gap stabilization, so that optimal policy does not take the relatively trivial form of completely stabilizing the predictable components of both variables. A special case is when the cost-push disturbance is an $\mathrm{AR}(1)$ process,

$$
u_{t+1}=\rho u_{t}+\varepsilon_{t+1},
$$

where $0 \leq \rho<1$ and $\varepsilon_{t+1}$ is an exogenous iid shock. ${ }^{14}$

The forward-looking aggregate-demand (IS) equation takes the form

$$
x_{t+1}=x_{t+2 \mid t}-\sigma\left(i_{t+1 \mid t}-\pi_{t+2 \mid t}-r_{t+1}^{n}\right),
$$

where $i_{t}$, the "instrument rate," is a short nominal interest rate and the central bank's instrument, $\sigma$ is a positive coefficient (the intertemporal elasticity of substitution), and $r_{t+1}^{n}$ is an

\footnotetext{
13 Which interpretation we take has no consequences for our analysis of optimal policy, since the surprise component of inflation makes in any event only an exogenous and constant contribution to the expected losses computed below.

${ }^{14}$ Here we assume that the same shock $\varepsilon_{t+1}$ represents both the surprise component of inflation in period $t+1$ and the innovation in period $t+1$ in the distortion $u_{t+2 \mid t+1}$ that affects "voluntary" inflation in period $t+2$. These could be the same process, if, for example, both are due to exogenous variation in the retail markup. More generally, however, all that really matters for our subsequent analysis is that the forecastable component $u_{t+1 \mid t}$ is assumed to be an AR(1) process. Allowing a "surprise inflation" term that is independent of this process makes no difference for our conclusions.
} 
exogenous disturbance. Again, conditional expectations are taken one period earlier than in the standard Euler equation, because interest-sensitive private expenditure is assumed to be predetermined for one period. This "time to plan" (argued in Christiano and Vigfusson [6] and Edge [8] to be realistic at least in the case of investment spending) is included in order to obtain the implication that monetary policy changes have no effect upon output, either, during the period of the change. Again, we allow for a "surprise" component of output, which may be interpreted as exogenous variation in some other component of aggregate expenditure, such as government purchases, that are not predetermined.

The forecastable component of the disturbance process, $r_{t+1 \mid t}^{n}$, represents exogenous variation in the Wicksellian "natural" (real) rate of interest, the real interest rate consistent with a zero output gap. This represents a composite of disturbances that affect the desired timing of expenditure and disturbances that affect potential output, since our IS equation is written in terms of the output gap rather than output. ${ }^{15}$ As long as our stabilization objectives can be defined in terms of inflation and the output gap (rather than output directly), only the effect of such factors upon the natural rate of interest matters for our analysis. A special case is when the natural rate of interest is an $\mathrm{AR}(1)$ process,

$$
r_{t+1}^{n}=\bar{r}+\omega\left(r_{t}^{n}-\bar{r}\right)+\eta_{t+1},
$$

where $0 \leq \omega<1, \bar{r}$ is the average natural real rate and $\eta_{t+1}$ is an exogenous iid shock in period $t+1 .^{16}$

The inclusion of the decision lags in our structural relations implies that inflation and the output gap fulfill

$$
\begin{aligned}
& \pi_{t+1}=\pi_{t+1 \mid t}+u_{t+1}-u_{t+1 \mid t}, \\
& x_{t+1}=x_{t+1 \mid t}+\sigma\left(r_{t+1}^{n}-r_{t+1 \mid t}^{n}\right),
\end{aligned}
$$

so that both inflation and the output gap are determined one period in advance, up to "surprise" terms that are completely exogenous. Thus, policy should be aimed solely at influencing the evolution of the forecastable components of inflation and the output gap, the private sector's inflation and output-gap "plans", $\pi_{t+1 \mid t}$ and $x_{t+1 \mid t}$. Thus, taking the expectation in period $t$ of (2.1) and (2.3), we can interpret them as describing how private-sector plans in period $t$ for

\footnotetext{
${ }^{15}$ See Woodford [47, chapter 4], for discussion of how various types of real disturbances affect this variable.

${ }^{16}$ Once again, it does not necessarily make sense to equate the "surprise" component of the output gap with the innovation in the natural rate, but this notational economy does not affect any of our subsequent conclusions.
} 
inflation and the output gap in period $t+1, \pi_{t+1 \mid t}$ and $x_{t+1 \mid t}$, are determined by expectations of: (1) inflation and the output gap in period $t+2, \pi_{t+2 \mid t}$ and $x_{t+2 \mid t},(2)$ the interest rate in period $t+1, i_{t+1 \mid t}$, and (3) the cost-push shock and natural interest rate in period $t+1, u_{t+1 \mid t}$ and $r_{t+1 \mid t}^{n}$. This modification of the basic model thus emphasizes, in equation (2.3), that monetary policy affects the economy not through the value set for the current short interest rate but rather by the expectations created regarding future interest rates. ${ }^{17}$ Actual inflation and the output gap in period $t+1$ are then determined by (2.5) and (2.6).

It follows from this last observation that there is no reason for surprise variations in the short-term interest rate to ever be chosen by the central bank. Such surprises can have no advantages in terms of improved stabilization of inflation or output, and if there is even a tiny degree of preference for less interest-rate variability (for reasons such as those discussed in Woodford [47, chapter 6]), it will therefore be optimal to make the interest rate perfectly forecastable one period in advance. We shall therefore restrict our attention to decision-making procedures under which the central bank's instrument is predetermined. One way to ensure this is for the central bank to make a decision in period $t$, denoted $i_{t+1, t}$, regarding the interest rate to be set in period $t+1$; several of the policy frameworks considered below incorporate this feature. This illustrates the more general point that a desirable decisionmaking framework may require the bank to decide, during the period- $t$ decision cycle, about matters in addition to the current setting of its instrument $i_{t}$.

We assume an intertemporal social loss function of the form

$$
\mathrm{E} \sum_{t=t_{0}}^{\infty} \beta^{t-t_{0}} L_{t}
$$

the expected value of the sum of discounted future period losses, starting in an arbitrary initial period $t_{0}$. (The question of the information with respect to which it is appropriate to condition in evaluating alternative policies is considered below.) The period losses are given by a period loss function of the form

$$
L_{t}=\frac{1}{2}\left[\pi_{t}^{2}+\lambda\left(x_{t}-x^{*}\right)^{2}\right]
$$

where $\lambda$ is the nonnegative relative weight on output-gap stabilization, and $x^{*}$ is the socially optimal output gap (for simplicity assumed constant), which is positive if potential output on

\footnotetext{
${ }^{17}$ This is also largely the case in the standard model, as is emphasized in Rotemberg and Woodford [28] and Woodford [44], since expected future interest rates enter indirectly via the expectations of future inflation and output gaps that enter equations (2.1) and (2.3).
} 
average, due to some distortion, falls short of the socially optimal output level. ${ }^{18}$ The discount factor $\beta$ in (2.7) is assumed to be the same as the coefficient appearing in (2.1). Woodford [47, chapter 6], shows that this form of loss function can be derived as a quadratic approximation to the (negative of) expected utility of the representative household in the same optimizing sticky-price model as is used to derive structural relations (2.1) and (2.3). And apart from this, it is a commonly assumed representation of the objectives of a central bank engaged in "flexible inflation targeting" (for instance, King [16] and Svensson [36]).

We assume that the private sector and the central bank have the same information. Specifically, we assume that both observe the current realization $u_{t}$ in period $t$, and have the same information in period $t$ about the future evolution of the exogenous disturbances; thus, for example, the private sector's conditional expectation $u_{t+\tau \mid t}$, regarding any period $\tau>0$, is assumed to also be the expectation regarding that exogenous variable conditional upon the central bank's information during its period- $t$ decision cycle. We also assume that any random element in the central bank's period- $t$ decisions is revealed to the private sector in period $t$. The only asymmetry is that in our discussion of specific central bank decision procedures, we assume that the central bank makes its period- $t$ decisions (such as its commitment $i_{t+1, t}$ ) without being able to observe the values of period- $t$ forward-looking variables, such as private-sector plans $\pi_{t+1 \mid t}$ and $x_{t+1 \mid t}$. This allows us to avoid the circularity of supposing that the central bank can directly respond in period $t$ to forward-looking variables that themselves depend upon the central bank's period- $t$ decisions. However, in a rational-expectations equilibrium, the period- $t$ forward-looking variables will be functions of the current values of predetermined and exogenous variables (about which the bank and the private sector have the same information), and thus the bank has sufficient information to allow it to perfectly forecast the period- $t$ variables that it does not directly observe. We also compute the equilibria associated with alternative central-bank decision procedures on the assumption that these procedures are perfectly understood by the private sector; this includes a correct understanding by the private sector of the central bank's model of the economy, insofar as this model is used in the bank's decisions. When the bank's model matters, we assume that it is the same as the true model of the economy (described by equations (2.1) and (2.3) and the stochastic processes governing the exogenous disturbances, (2.2) and (2.4) in the special case), which is to say, the model with which private-sector expectations are assumed

\footnotetext{
${ }^{18}$ Note that time variation in the optimal output gap has been allowed for by the inclusion of the "cost-push" disturbance term in (2.1). Following prior literature, we separately consider the consequences of a non-zero mean distortion and the consequences of random variation in the distortion.
} 
to be consistent.

The model assumed here, while familiar, has some features that are worthy of comment. Both the AS and IS equations incorporate important forward-looking elements. In particular, the tradeoff that the central bank faces in period $t$ between alternative values for the forecastable components of inflation and the output gap in period $t+1\left(\pi_{t+1 \mid t}\right.$ and $x_{t+1 \mid t}$ respectively) depends upon private-sector expectations regarding equilibrium in still later periods (due to the $\pi_{t+2 \mid t}$ term in (2.1), and hence upon expectations regarding future policy. This gives rise to a "conditional" or "stabilization bias" in the responses to shocks resulting from discretionary optimization, as we show explicitly below.

Indeed, our simple model is extremely forward-looking, in that the equations that determine $\pi_{t+\tau \mid t}$ and $x_{t+\tau \mid t}$ for all $\tau>0$ involve no other variables, except period- $t$ expectations regarding future central bank actions $i_{t+\tau \mid t}$ and regarding the evolution of the exogenous disturbances $u_{t+\tau \mid t}, r_{t+\tau \mid t}^{n}$. This means a purely forward-looking decision procedure for monetary policy - one that depends simply upon the central bank's forecasts in period $t$ of the future evolution of its target variables - will result in period- $t$ decisions that depend only upon period- $t$ expectations regarding the evolution of the exogenous disturbances, and not upon any current or lagged endogenous variables at all. ${ }^{19}$

This feature of our model is undoubtedly highly special, but it allows us to contrast the history-dependence that is required in order to implement optimal policy with the results of purely forward-looking procedures in an especially sharp way. In a more realistic model, many sorts of intrinsic dynamics would also likely be present, as a result of which lagged endogenous variables would matter for conditional forecasts of the future evolution of the target variables. But our general points about the generic inefficiency of purely forward-looking procedures would remain valid; the quantitative significance of the inefficiency in more complex, but more realistic, models remains a topic for future research.

\footnotetext{
${ }^{19}$ An advantage of our allowance for one-period decision lags in both spending and pricing decisions is that feedback from even the current quarter's inflation rate and output gap, as in the rule proposed by Taylor [40], is here clearly an example of dependence upon variables that are irrelevant under a purely forward-looking procedure. This allows us a sharp contrast between purely prospective procedures, such as those often recommended in the literature on inflation targeting, and purely backward-looking rules such as the "Taylor rule." We believe that this feature of our model is quite realistic (assuming the "period" to be a typical length of time between central bank decision cycles), and thus worth the minor complication involved. In fact, inflation and output may be largely predetermined for significantly longer periods of time.
} 


\subsection{Optimal equilibrium responses to shocks}

By an equilibrium of this model, we mean a triple of stochastic processes for inflation, the output gap and the interest rate that satisfy equations (2.1) and (2.3). Note that our concept of "equilibrium" does not include any assumption that the central bank behaves optimally, as our task is in fact to investigate the equilibria associated with alternative candidate policy-making procedures on the part of the central bank.

We first consider the equilibrium from some period $t_{0}$ onward that is optimal in the sense of minimizing (2.7). In this calculation, the expectation is conditional upon the state of the world in period $t_{0}$, denoted $\mathrm{E}_{t_{0}}$, when we imagine being able to choose among equilibria that remain possible from that period onward. Let us call this " $t_{0}$-optimality"; it corresponds to the type of optimal plan with which the literature on dynamic Ramsey taxation, for example, is typically concerned. (We shall subsequently also define optimality from a "timeless perspective" that we shall argue is more appropriate when choosing among policy rules.)

We begin by observing that, conditional upon information available one period in advance, the period- $t+1$ loss function may be written

$$
\begin{aligned}
\mathrm{E}_{t}\left[L_{t+1}\right] & =\frac{1}{2} \mathrm{E}_{t}\left[\pi_{t+1 \mid t}^{2}+\lambda\left(x_{t+1 \mid t}-x^{*}\right)^{2}\right]+\frac{1}{2} \mathrm{E}_{t}\left[\left(\pi_{t+1}-\pi_{t+1 \mid t}\right)^{2}+\lambda\left(x_{t+1}-x_{t+1 \mid t}\right)^{2}\right] \\
& =\frac{1}{2} \mathrm{E}_{t}\left[\pi_{t+1 \mid t}^{2}+\lambda\left(x_{t+1 \mid t}-x^{*}\right)^{2}\right]+\frac{1}{2} \mathrm{E}_{t}\left[\left(u_{t+1}-u_{t+1 \mid t}\right)^{2}+\lambda \sigma^{2}\left(r_{t+1}^{n}-r_{t+1 \mid t}^{n}\right)^{2}\right],
\end{aligned}
$$

using (2.5) and (2.6). The second term on the right-hand side of the second line is independent of policy, as it depends only upon the exogenous disturbance processes. Thus (using also the fact that $\mathrm{E}_{t_{0}} L_{t+1}=\mathrm{E}_{t_{0}}\left[\mathrm{E}_{t} L_{t+1}\right]$ for all $\left.t \geq t_{0}\right)$, we may replace each term of the form $\mathrm{E}_{t_{0}} L_{t+1}$ in $(2.7)$ by the conditional expectation of the first term on the right-hand side above, plus a positive constant. Since the initial term $\mathrm{E}_{t_{0}} L_{t_{0}}$ is also independent of policy (given predetermined initial values for $\pi_{t_{0} \mid t_{0}-1}$ and $x_{t_{0} \mid t_{0}-1}$ ), our problem may equivalently be defined as that of choosing paths for the forecastable components of inflation and the output gap, the private-sector oneperiod-ahead plans for inflation and the output gap, $\left\{\pi_{t+1 \mid t}\right\}_{t=t_{0}}^{\infty}$ and $\left\{x_{t+1 \mid t}\right\}_{t=t_{0}}^{\infty}$, so as to minimize

$$
\mathrm{E}_{t_{0}} \sum_{t=t_{0}}^{\infty} \beta^{t+1-t_{0}} \frac{1}{2}\left[\pi_{t+1 \mid t}^{2}+\lambda\left(x_{t+1 \mid t}-x^{*}\right)^{2}\right] .
$$

Note that once we have determined the optimal paths for the forecastable components, we shall have determined the optimal paths for inflation and the output gap as well, because of (2.5) and $(2.6)$ 
We thus need ask only what constraints the equilibrium relations (2.1) and (2.3) impose upon the possible paths of the forecastable components of these two variables. One such constraint is

$$
\pi_{t+1 \mid t}=\beta \pi_{t+2 \mid t}+\kappa x_{t+1 \mid t}+u_{t+1 \mid t}
$$

obtained by taking the conditional expectation of (2.1) one period in advance. This is in fact the only constraint. For given any processes for the forecastable components satisfying (2.9), the inflation processes implied by (2.5) then necessarily satisfies (2.1); and given any processes for inflation and the output gap, one can solve (2.3) for a forecastable interest-rate process $\left\{i_{t+1 \mid t}\right\}_{t=t_{0}}^{\infty}$ that satisfies that condition as well.

Thus, we form the Lagrangian

$$
\mathcal{L}_{t_{0}} \equiv \mathrm{E}_{t_{0}} \sum_{t=t_{0}}^{\infty} \beta^{t+1-t_{0}}\left\{\begin{array}{l}
\frac{1}{2}\left[\pi_{t+1 \mid t}^{2}+\lambda\left(x_{t+1 \mid t}-x^{*}\right)^{2}\right] \\
+\Xi_{t+1}\left[\beta \pi_{t+2 \mid t}+\kappa x_{t+1 \mid t}+u_{t+1 \mid t}-\pi_{t+1 \mid t}\right]
\end{array}\right\}
$$

where $\Xi_{t+1}$ is the Lagrange multiplier associated with the constraint $(2.9) .{ }^{20}$ We note that $\Xi_{t+1}$ depends on period- $t$ information only. Differentiating with respect to $\pi_{t+1 \mid t}$ and $x_{t+1 \mid t}$ for any $t \geq t_{0}$ gives the first-order conditions

$$
\begin{aligned}
\pi_{t+1 \mid t}-\Xi_{t+1}+\Xi_{t} & =0 \\
\lambda\left(x_{t+1 \mid t}-x^{*}\right)+\kappa \Xi_{t+1} & =0
\end{aligned}
$$

for all $t \geq t_{0}$, with the initial condition

$$
\Xi_{t_{0}}=0
$$

We eliminate $\Xi_{t}$ from (2.11) and (2.12) and get the consolidated first-order condition

$$
\pi_{t+1 \mid t}+\frac{\lambda}{\kappa}\left(x_{t+1 \mid t}-x_{t \mid t-1}\right)=0
$$

for $t>t_{0}$, and

$$
\pi_{t+1 \mid t}+\frac{\lambda}{\kappa}\left(x_{t+1 \mid t}-x^{*}\right)=0
$$

for $t=t_{0}$.

In order to determine the stochastic processes for $\pi_{t+1 \mid t}$ and $x_{t+1 \mid t}$, we use (2.14) and (2.15) to eliminate $\pi_{t+1 \mid t}$ and $\pi_{t+2 \mid t}$ in (2.9). For $\lambda>0$, this yields a second-order difference equation for $x_{t+1 \mid t}$ for $t \geq t_{0}$,

$$
x_{t+2 \mid t}-2 a x_{t+1 \mid t}+\frac{1}{\beta} x_{t \mid t-1}=\frac{\kappa}{\beta \lambda} u_{t+1 \mid t},
$$

\footnotetext{
${ }^{20}$ Relative to the formulation in Woodford [45], the Lagrange multiplier is defined with the opposite sign, so as to be interpreted as marginal losses rather than gains.
} 
where

$$
2 a \equiv 1+\frac{1}{\beta}+\frac{\kappa^{2}}{\beta \lambda}
$$

and (2.13) and (2.15) give rise to an initial condition,

$$
x_{t_{0} \mid t_{0}-1} \equiv x^{*}
$$

where we emphasize that the notation $x_{t_{0} \mid t_{0}-1}$ is here temporarily used only to introduce the initial condition (2.18) in (2.16), corresponding to the initial condition (2.13), rather than to denote the one-period-ahead output-gap plan in period $t_{0}-1$. The characteristic equation,

$$
\mu^{2}-2 a \mu+\frac{1}{\beta}=0
$$

has two roots (eigenvalues of the dynamic system), $c \equiv a-\sqrt{a^{2}-1 / \beta}$ and $1 /(\beta c)$, such that $0<c<1<1 / \beta<1 /(\beta c)$. Then, by standard methods, the solution can be written

$$
x_{t+1 \mid t}=-\frac{\kappa}{\lambda} c \sum_{j=0}^{\infty}(\beta c)^{j} u_{t+1+j \mid t}+c x_{t \mid t-1}
$$

for $t \geq t_{0}$.

Under the assumption $(2.2)$, the term $\sum_{j=0}^{\infty}(\beta c)^{j} u_{t+1+j \mid t}$ is given by $\rho u_{t} /(1-\beta \rho c)$, and $(2.20)$ becomes

$$
\begin{aligned}
x_{t+1 \mid t} & =-\frac{\kappa}{\lambda} \frac{\rho c}{1-\beta \rho c} u_{t}+c x_{t \mid t-1} \\
& =-\frac{\kappa}{\lambda} \frac{\rho c}{1-\beta \rho c} \sum_{j=0}^{t-t_{0}} c^{j} u_{t-j}+c^{t+1-t_{0}} x^{*},
\end{aligned}
$$

where the last step uses (2.18). Given this solution for $x_{t+1 \mid t}$, we can then use (2.14) to find equilibrium values of $\pi_{t+1 \mid t}$. We thus obtain

$$
\begin{aligned}
\pi_{t+1 \mid t} & =\frac{\rho c}{1-\beta \rho c} u_{t}+\frac{\lambda}{\kappa}(1-c) x_{t \mid t-1} \\
& =\frac{\rho c}{1-\beta \rho c}\left[u_{t}-(1-c) \sum_{j=1}^{t-t_{0}} c^{j-1} u_{t-j}\right]+\frac{\lambda}{\kappa}(1-c) c^{t-t_{0}} x^{*}
\end{aligned}
$$

again simplifying by assuming (2.2).

For $\lambda=0$, we directly have the simple solution

$$
\begin{aligned}
x_{t+1 \mid t} & =-\frac{1}{\kappa} u_{t+1 \mid t}, \\
\pi_{t+1 \mid t} & =0
\end{aligned}
$$

to $(2.14)$ and (2.15). Since $c \rightarrow 0$ when $\lambda \rightarrow 0$, this can be shown to be the limit of (2.21)-(2.24). 


\subsection{Optimality from a "timeless perspective"}

This equilibrium, however, specifies inflation and output-gap processes that depend upon how long it has been since the period $t_{0}$ in which the " $t_{0}$-optimal" equilibrium was chosen. Obviously, exactly the same criterion would lead one to choose a different equilibrium in some later period, rather than the continuation of the equilibrium chosen as optimal in period $t_{0}$. This is just the familiar problem of time-inconsistency of optimal plans in problems of this kind, first identified by Kydland and Prescott [18]. Formally, it results from the fact that initial condition (2.13) is specified for period $t_{0}$, though the solution generally involves $\Xi_{t} \neq 0$ in later periods.

What this means, intuitively, is that the proposed criterion for optimality allows one to select an equilibrium from period $t_{0}$ onward that exploits the fact that private-sector expectations in earlier periods are already given when the paths from $t_{0}$ onward are chosen. This allows one to choose a "surprise" inflation for "just this once" while committing never to do so again, as one would suffer all of the consequences of anticipated inflation if one chose an equilibrium in which inflation is planned for a period well after $t_{0}$. Of course, if one allows oneself to exploit preexisting expectations in this way, it would be equally appealing to allow "one last unexpected inflation" in some later period as well. This is the reason for the time-inconsistency of optimal policy in this sense.

It therefore makes sense not to demand of a monetary policy rule that commitment to it from some date $t_{0}$ onward be expected to implement an equilibrium that is " $t_{0}$-optimal". Instead, we consider optimality from the "timeless perspective" recommended by Woodford [45] and Giannoni and Woodford [11]. A policy rule is optimal from a timeless perspective if (i) it has a time-invariant form, and (ii) commitment to the rule from any date $t_{0}$ onward determines an equilibrium that is optimal, subject to at most a finite number of constraints on the initial evolution of the endogenous variables. Regarding constrained optimality as sufficient weakens the sense in which the rule is required to be optimal; but there may be no time-invariant policy that would be optimal in an unconstrained sense (that is, that would be $t_{0}$-optimal). Furthermore, the fact that the economy's expected evolution under commitment to the rule is optimal subject only to a constraint on its short-run evolution (and not, for example, any constraint that requires long-run outcomes to resemble short-run outcomes) means that the constraints on short-run outcomes are ones that an optimizing central bank would wish to be subject to - and in particular, would wish for the private sector to expect it to be subject to - in

the future. Acceptance of such a constraint thus means conformity to a rule of behavior to which 
it would have been optimal to commit oneself in the past. Acting in conformity with such a rule is a way of making it more credible that one will also act in conformity with it in the future, and the central bank has an interest in creating the latter expectation. Note that a policy rule that satisfies this criterion in period $t_{0}$ will also satisfy it if the matter is reconsidered in any later period; thus this approach to policy choice eliminates the problem of time-inconsistency. ${ }^{21}$

The definition just given does not identify the constraints on the economy's short-run evolution that should be accepted, and so there need not be a unique state-contingent evolution from date $t_{0}$ onward that can qualify as optimal from a timeless perspective. Nonetheless, the constraints on the initial evolution of the economy are not arbitrary, for most constraints on short-run outcomes have the property that even if one is subject to them, it would be optimal to choose an equilibrium that does not satisfy them in the future. The requirement that the equilibrium chosen be implementable through commitment to a time-invariant policy imposes a strong self-consistency requirement on the choice of the initial constraints, though it does not uniquely determine them. In fact, in a linear-quadratic policy problem of the kind considered here (or in Giannoni and Woodford [11]), all policy rules that are optimal from a timeless perspective lead to the same long-run average values of endogenous variables such as output and inflation, and to the same equilibrium responses to unexpected shocks that occur at date $t_{0}$ or later. The equilibria that are implemented by these rules differ only in a transitory, deterministic component of the equilibrium paths of variables like inflation and output.

In the example considered here, a rule that is optimal from a timeless perspective must bring about an equilibrium from date $t_{0}$ onward that minimizes $(2.7)$, subject to the constraints that (2.1) and (2.3) hold for each $t \geq t_{0}$, and the additional constraint

$$
\pi_{t_{0}+1 \mid t_{0}}=\bar{\pi}_{t_{0}}
$$

where the constraint value $\bar{\pi}_{t_{0}}$ is selected in a time-invariant way, as a function of the economy's state in period $t_{0}$ (after the realization of the exogenous disturbances, but before the determination of the endogenous variables). Furthermore, the rule for selecting $\bar{\pi}_{t_{0}}$ must be one that is satisfied by $\pi_{t+1 \mid t}$ for all $t>t_{0}$ in the constrained-optimal equilibrium from the standpoint of period $t_{0}$. Here we give two examples of rules for selecting the constraint on short-run outcomes

\footnotetext{
${ }^{21}$ Of course, this property alone does not eliminate the incentive to deviate from such a policy commitment in order to reduce expected losses conditional upon the state of the world at the time of the contemplated deviation. We do not here attempt to model the mechanism that makes it possible for a central bank to commit itself to a decision procedure other than unconstrained discretionary optimization. However, even granting the possibility of commitment, it remains more credible that an institution should feel bound by a past commitment when the logic of its own past analysis does not itself justify deviation at a later date.
} 
that have the desired property; this will suffice both to show that it is possible to satisfy the self-consistency requirement, and to illustrate the point that the constraint need not be uniquely defined..$^{22}$

We first observe that if a $t_{0}$-optimal equilibrium has been chosen at a date $t_{0}$ that is now infinitely far in the past, equations (2.22) and (2.24) reduce to

$$
\begin{aligned}
x_{t+1 \mid t} & =-\frac{\kappa}{\lambda} \frac{\rho c}{1-\beta \rho c} \sum_{j=0}^{\infty} c^{j} u_{t-j} . \\
\pi_{t+1 \mid t} & =\frac{\rho c}{1-\beta \rho c}\left[u_{t}-(1-c) \sum_{j=1}^{\infty} c^{j-1} u_{t-j}\right],
\end{aligned}
$$

This suggests one possible specification of a pair of constraints of the form (2.25): one requires that $\pi_{t_{0}+1 \mid t_{0}}$ satisfy $(2.27)$ for $t=t_{0}$. In fact, one easily sees that the evolution of expected inflation and output from date $t_{0}$ onward that minimizes (2.7) subject to this constraint is just the one that satisfies (2.26) and (2.27) for all $t \geq t_{0} \cdot{ }^{23}$ Hence, this is an example of a self-consistent constraint on the economy's short-run evolution of the kind discussed above. A time-invariant policy rule that yields the evolution (2.26) and (2.27) as a determinate equilibrium will therefore be optimal from a timeless perspective.

However, this is not the only state-contingent evolution from date $t_{0}$ onward that can be considered optimal from a timeless perspective. We may also select the constraints on short-run outcomes in a way that depends on the initial values of predetermined endogenous variables, rather than being a function solely of the history of exogenous disturbances as above. For example, suppose that in $(2.25)$ we use the value

$$
\bar{\pi}_{t_{0}}=\frac{\rho c}{1-\beta \rho c} u_{t_{0}}+\frac{\lambda}{\kappa}(1-c) x_{t_{0} \mid t_{0}-1}
$$

where $x_{t_{0} \mid t_{0}-1}$ here denotes the actual output-gap plan in period $t_{0}-1$. (Our choice of this specification of the initial condition is motivated by the observation that $\pi_{t_{0}+1 \mid t_{0}}$ would have to satisfy (2.23) in any $\tau$-optimal equilibrium chosen at a date $\tau<t_{0} \cdot{ }^{24}$ ) Under this specification,

\footnotetext{
${ }^{22}$ Giannoni and Woodford [11] provide a general approach to the choice of policy rules that are optimal from a timeless perspective, in the context of a broad class of linear-quadratic policy problems.

23 The problem reduces to finding a solution to the system consisting of (2.1) and (2.3) together with (2.11) and (2.12), with the initial condition (2.25) replacing (2.13). Our method of derivation of equations (2.27) and (2.26) makes it obvious that they satisfy all of these equations.

24 A generalization of the approach used here is developed in Giannoni and Woodford [11].
} 
the equilibrium that minimizes (2.7) subject to constraint (2.25) is given by

$$
\begin{aligned}
x_{t+1 \mid t} & =-\frac{\kappa}{\lambda} \frac{\rho c}{1-\beta \rho c} \sum_{j=0}^{t-t_{0}} c^{j} u_{t-j}+c^{t+1-t_{0}} x_{t_{0} \mid t_{0}-1} \\
\pi_{t+1 \mid t} & =\frac{\rho c}{1-\beta \rho c}\left[u_{t}-(1-c) \sum_{j=1}^{t-t_{0}} c^{j-1} u_{t-j}\right]+\frac{\lambda}{\kappa}(1-c) c^{t-t_{0}} x_{t_{0} \mid t_{0}-1}
\end{aligned}
$$

for all $t \geq t_{0}$.

The constraint (2.28) is observed to be self-consistent. For the solutions (2.29) and (2.30) imply (2.21) and (2.23) for any $t \geq t_{0}$. Hence, we find once again that a time-invariant rule that yields the evolution (2.29) and (2.30) as a determinate equilibrium is optimal from a timeless perspective.

For most values of the initial condition $x_{t_{0} \mid t_{0}-1}$, these state-contingent paths for expected inflation and expected output in (2.29) and (2.30) will be different from those in (2.26) and (2.27) (except asymptotically, when they coincide as $c^{t+1-t_{0}} \rightarrow 0$ ). They similarly both differ from the $t_{0}$-optimal equilibrium, described by (2.22) and (2.24), except asymptotically. However, both examples of a timelessly optimal equilibrium agree with one another, and with the $t_{0}$-optimal equilibrium, in the linear terms involving the exogenous disturbances in periods $t \geq t_{0}$. These several alternative conceptions of the optimal state-contingent evolution from period $t_{0}$ onward differ only in certain deterministic components of the equilibrium levels of inflation and output, that in each case become negligible for $t$ sufficiently greater than $t_{0}$.

The examples of timelessly optimal equilibria just discussed are only two of an infinite number of possibilities. More generally, we observe that the equilibrium resulting from adoption of a timelessly optimal policy rule must satisfy conditions (2.11) and (2.12) for all $t \geq$ $t_{0}$, for some value of $\Xi_{t_{0}}$. However, the value of $\Xi_{t_{0}}$ need not satisfy $(2.13)$ in general. Instead, $\Xi_{t_{0}}$ is selected as some function of the state of the world, denoted $h_{t_{0}-1}$, in the previous period. For future reference, we define the state of the world in period $t$ as $h_{t} \equiv$ $\left\{u_{t}, r_{t}^{n}, i_{t}, i_{t+1 \mid t}, \pi_{t+1 \mid t}, x_{t+1 \mid t} ; u_{t-1}, r_{t-1}^{n}, i_{t-1}, i_{t \mid t-1}, \pi_{t \mid t-1}, x_{t \mid t-1} ; \ldots\right\}$.

Our characterization of optimal equilibrium already allows us to reach one important conclusion about optimal policy. This is that a purely forward-looking decision procedure cannot be used to implement an optimal equilibrium. In the current model, the equations that determine the expected future values of the goal variables, $\pi_{t+\tau \mid t}$ and $x_{t+\tau \mid t}$ for $\tau \geq 1$, for any given expected future path of the central bank's instrument, depend only upon expectations in period $t$ of the future paths of the exogenous disturbances. Thus, if the central bank does not 
itself plan to condition its decisions in period $t$ or later on information other than information about the exogenous disturbance processes, then its forecasts of the future evolution of the target variables will be independent of any other information (specifically, the value of any lagged endogenous variables). Under a purely forward-looking decision procedure, its decisions during the period- $t$ decision cycle should similarly be independent of any such "irrelevant" information. And then, if a correct private-sector understanding of this policy rule results in a determinate rational-expectations equilibrium, the equilibrium will be one in which the evolution of the target variables is independent of "irrelevant" lagged endogenous variables. ${ }^{25}$

But we have seen that an optimal equilibrium is necessarily not of this kind. In the case that (2.2) and (2.4) hold, all information about the future evolution of the disturbances is summarized by the current disturbances $u_{t}$ and $r_{t}^{n}$. Thus, an equilibrium that could be implemented using a purely prospective decision procedure would have to make $\pi_{t+1 \mid t}$ and $x_{t+1 \mid t}$ functions of $u_{t}$ and $r_{t}^{n}$. Our above solutions do not have this character; instead, $x_{t \mid t-1}$ and, therefore, the entire history $\left\{u_{t-j}\right\}_{j=1}^{\infty}$, back at least to period $t_{0}$, affect the optimal values of both variables. Thus, a decision procedure that can implement an optimal equilibrium must involve a degree of historydependence not allowed for in the types of purely prospective policy procedures often assumed in discussions of inflation targeting. Examples of suitable sources of history-dependence are presented in sections 3 through 5 .

\subsection{Interest rates in an optimal equilibrium}

To each of the optimal paths for inflation and the output gap just characterized, there corresponds an optimal path for the nominal interest rate. Taking the conditional expectation of (2.3) in period $t$ and solving for $i_{t+1 \mid t}$, we obtain

$$
i_{t+1 \mid t}=r_{t+1 \mid t}^{n}+\pi_{t+2 \mid t}+\frac{1}{\sigma}\left(x_{t+2 \mid t}-x_{t+1 \mid t}\right) .
$$

Substitution of (2.14), which holds for all $t>t_{0}$ in a $t_{0}$-optimal equilibrium and in the equilibrium associated with any timelessly optimal policy rule, into (2.31) then yields

$$
i_{t+1 \mid t}=r_{t+1 \mid t}^{n}+\frac{\lambda \sigma-\kappa}{\lambda \sigma} \pi_{t+2 \mid t}
$$

\footnotetext{
${ }^{25}$ Even if equilibrium is indeterminate, if one expects that the equilibrium that should result in practice will be selected by a "minimum-state-variable" (MSV) criterion, like that suggested by McCallum [23], then the equilibrium selected will not depend upon the "irrelevant" lagged endogenous variables, and the argument in the text goes through. If one admits that non-MSV equilibria may occur, then the equilibria that may occur will include a large number of equilibria other than the optimal one.
} 
for all $t>t_{0}$. Finally, substitution of the equilibrium values of $\pi_{t+2 \mid t}$ discussed above yields a stochastic process for the forecastable component of the interest rate.

For example, in the case of a timelessly optimal policy resulting in the equilibrium described by (2.26) and (2.27), the associated forecastable component of the interest rate is given by $i_{t+1 \mid t}=i_{t+1}^{*}$, where

$$
i_{t+1}^{*} \equiv \bar{r}+\omega\left(r_{t}^{n}-\bar{r}\right)+\frac{\lambda \sigma-\kappa}{\lambda \sigma} \frac{\rho c}{1-\beta \rho c}\left[\rho u_{t}-(1-c) \sum_{j=0}^{\infty} c^{j} u_{t-j}\right] .
$$

(Here we also assume (2.4), allowing us to replace $r_{t+1 \mid t}^{n}$ by $\bar{r}+\omega\left(r_{t}^{n}-\bar{r}\right)$.) Note that the exogenous process $\left\{i_{t+1}^{*}\right\}_{t=t_{0}}^{\infty}$ also indicates how the expected interest rate must evolve, as a function of the history of exogenous disturbances, in any optimal equilibrium that has been in existence for a long enough period of time.

Alternatively, in the case of a timelessly optimal policy resulting in the equilibrium described by $(2.21)$ and $(2.23)$, the expected interest rate is given by $i_{t+1 \mid t}=\bar{\imath}_{t+1}$, where

$$
\bar{\imath}_{t+1} \equiv \bar{r}+\omega\left(r_{t}^{n}-\bar{r}\right)+\frac{\lambda \sigma-\kappa}{\lambda \sigma} \frac{\rho c}{1-\beta \rho c}(\rho+c-1) u_{t}+f x_{t \mid t-1},
$$

where

$$
f \equiv \frac{\lambda \sigma-\kappa}{\kappa \sigma}(1-c) c .
$$

Note that in (2.33) we have expressed the endogenous process $\bar{\imath}_{t+1}$ as a time-invariant function of the state of the world $h_{t}$, a representation that will be useful for our discussion below of associated reaction functions; a corresponding expression for $i_{t+1 \mid t}$ as a function of $h_{t_{0}-1}$ and the exogenous disturbances in periods $t_{0}$ through $t$ can be obtained by substituting expression (2.29) for $x_{t \mid t-1}$ into (2.33). And once again, we observe that, if initial conditions $h_{t_{0}-1}$ are consistent with the stationary optimal equilibrium (2.26) and (2.27), processes (2.32) and (2.33) will coincide exactly at all times. (This can be seen by observing that if one instead uses (2.26) to substitute for $x_{t \mid t-1}$ in (2.33), one obtains (2.32).)

None of our optimality conditions place any restrictions upon the path of the unforecastable component of the interest rate, and indeed, from the point of view of the objective assumed above, its path is completely arbitrary, as it has no effect upon either spending or pricing decisions in this model. However, it is plausible to assume that one should prefer less variable interest rates, other things being equal. ${ }^{26}$ It follows that it can never be desirable to have any

\footnotetext{
${ }^{26}$ Woodford [47, chapter 6] discusses reasons why one may even be willing even to accept somewhat more variable inflation and output gaps for the sake of improved interest-rate stabilization. Svensson [38, section 5.6] expresses scepticism about those reasons. We abstract from such concerns here, in order to simplify the algebra in our analysis.
} 
unforecastable interest-rate fluctuations; thus we stipulate that an optimal policy will imply that $i_{t+1}=i_{t+1 \mid t}$ at all times. With this additional stipulation, we can now derive unique equilibrium interest-rate processes associated with each of the possible optimal equilibria. These are given by the above equations, with $i_{t+1}$ replacing $i_{t+1 \mid t}$.

This result still only tells us how it is desirable for interest rates to evolve in equilibrium, as a function of the disturbances that hit the economy; it does not tell us what form of policy rule should be adopted by the central bank, in order to bring about an equilibrium of the desired character. Simply committing to set interest rates as the specified function of the history of disturbances is not the only type of policy rule that would be consistent with an equilibrium of the desired kind, and in fact we shall argue that this would not be a desirable approach to the implementation of optimal policy - it would be inferior to other approaches, both on the ground of non-robustness of the policy rule to changes in the model of the economy, and on the ground that equilibrium will not be determinate under such a rule.

Still, this characterization of optimal equilibrium interest-rate paths can help to identify possible forms of policy rules that will be consistent with one or another of the optimal equilibria just discussed. In particular, any given explicit decision procedure will imply a reaction function

$$
i_{t+1}=F\left(s_{t+1}, h_{t}\right)
$$

indicating the way in which the central bank's instrument is set as a function of the information available to it in decision cycle $t+1$, consisting of all exogenous disturbances, $s_{t+1} \equiv\left(u_{t+1}, r_{t+1}^{n}\right)$, in period $t+1$ and the state of the world, $h_{t}$, in period $t .^{27}$ Recall that we assume that all exogenous disturbances $s_{t+1}$ realized in period $t+1$ are already known to the central bank before its instrument setting for period $t+1$ must be chosen, but that period- $t+1$ endogenous variables, the inflation and output-gap plans $\pi_{t+1 \mid t}$ and $x_{t+1 \mid t}$, that generally depend upon the bank's action, cannot be directly responded to; instead the bank can respond only to its forecasts of how these variables should evolve. However, all elements of $h_{t}$, including period- $t$ endogenous variables, are assumed to be public information prior to the bank's period- $t+1$ decision cycle; thus $i_{t+1}$ may respond to them.

In this study we shall restrict our attention to decision procedures of two broad types, targeting rules and explicit instrument rules. Each of these classes implies a further restriction upon the possible form of the reaction function. In the case of a targeting rule, the setting of $i_{t}$

\footnotetext{
${ }^{27}$ In general, the vector $s_{t+1}$ includes all information as of period $t+1$ about the paths of the exogenous disturbances in periods $t+\tau$ for $\tau \geq 1$. In the special case that both disturbances are Markovian, as assumed in (2.2) and (2.4), the vector $s_{t+1}$ has only two elements, $u_{t+1}$ and $r_{t+1}^{n}$.
} 
chosen during the period- $t$ decision cycle is not expected to affect the period- $t$ target variables, $\pi_{t}$ and $x_{t}$, since these are assumed to be predetermined; only the private sector's forecast of the setting during previous periods matters for the period- $t$ target variables. Hence, the targeting procedure must instead be used to choose a commitment $i_{t+1, t}$ regarding the interest-rate setting to be adopted in the following period; the interest rate itself is simply set in accordance with the commitment made during the previous decision cycle: $i_{t+1}=i_{t+1, t}$. It then follows that under any such rule, the interest rate $i_{t+1}$ will be a function of information available to the central bank during its period-t decision cycle. Under our information specification, this means a function of variables that are predetermined in period $t$, or exogenous variables realized in period $t$, so that the implied reaction function associated with such a policy must be of the more restricted form

$$
i_{t+1}=F\left(s_{t}, h_{t-1}\right) .
$$

Given that the reaction function must have the form (2.36), we can uniquely identify the implied reaction function that must be implied by any targeting rule that is consistent with a particular equilibrium from the adoption date $t_{0}$ onward. To do this, we simply read off our solution, above, for $i_{t+1}$ as a function of $s_{t}$ and $h_{t-1}$. Thus, a targeting rule consistent with the equilibrium (2.26) and (2.27) must yield the implied reaction function

$$
i_{t+1}=i_{t+1}^{*}
$$

where $i_{t+1}^{*}$ is defined in (2.32), while a targeting rule consistent with the equilibrium (2.21) and (2.23) must yield the implied reaction function

$$
i_{t+1}=\bar{\imath}_{t+1}
$$

where $\bar{\imath}_{t+1}$ is defined in (2.33). Of course, these reaction functions do not yet uniquely identify the form of the policy rule; alternative high-level policy prescriptions might imply the same reaction function. We give examples below of targeting procedures that imply each of these reaction functions.

In the case of an explicit instrument rule, instead, the policy rule is just a commitment to set the instrument in accordance with a particular reaction function. One advantage of this way of specifying the policy rule is that the instrument setting in period $t+1$ need no longer be a function solely of information available at the time of the period- $t$ decision cycle; it can instead make use of information available only by the time of the period- $t+1$ decision cycle. 
Because unforecastable interest-rate movements are undesirable, an optimal instrument rule will nevertheless necessarily be of the restricted form

$$
i_{t+1}=F\left(h_{t}\right)
$$

rather than of the form (2.35). Yet there remains an advantage of family (2.39) over the even more restrictive family $(2.36)$, which is that it allows $i_{t+1}$ to respond to endogenous variables realized in period $t$-information that we assume is available to the private sector when making its period- $t$ decisions, but not during the central bank's period- $t$ decision cycle. This can be useful in that it allows the central bank to respond in period $t+1$ to private-sector decisions in period $t, \pi_{t+1 \mid t}$ and $x_{t+1 \mid t}$, that are inconsistent with the equilibrium that it is trying to bring about (and thus inconsistent with its own forecasts of those variables during its period- $t$ decision cycle). A commitment to such responses can be useful, as we show later, in excluding unwanted alternative rational-expectations equilibria.

In the case of the more flexible specification (2.39), we can no longer uniquely determine the reaction function from our above solution for the equilibrium interest-rate process. Our discussion above allowed us to determine how $i_{t+1}$ must depend upon $s_{t}$ and $h_{t-1}$ in the equilibrium that we wish to implement. However, many endogenous variables in $h_{t}$ will also be functions of these variables, and (assuming that the variables co-move as in the desired equilibrium) the desired variation in interest rates can therefore be arranged by setting $i_{t+1}$ as a function of these variables, rather than by setting it as a direct function of the variables observed by the central bank by the time of its period- $t$ decision cycle. There will thus generally be a large number of possible instrument rules consistent with a given equilibrium, even though there is a one-to-one correspondence between instrument rules and reaction functions.

\subsection{The problem of indeterminacy}

One aspect of the problem of implementing optimal policy is finding a decision procedure that is consistent with an optimal equilibrium, as characterized above. But even when we find a procedure that satisfies this criterion - say, a targeting rule that implies reaction function (2.37) or (2.38) - there remains the question whether the optimal equilibrium is the only equilibrium consistent the specified policy rule. In addressing this question, it suffices to characterize a policy rule in terms of the reaction function that it implies. ${ }^{28}$ Our question is then whether the

\footnotetext{
${ }^{28}$ Note, however, that for some other questions - notably the analysis of robustness - the reaction function is not a sufficient description of a policy rule. It is for this reason that we are careful in this paper not to identify policy rules with their implied reaction functions.
} 
system of equations consisting of (2.1), (2.3) and either (2.36) or (2.39) has a unique bounded (or non-explosive) rational-expectations equilibrium. ${ }^{29}$ In this case, we shall say that equilibrium is determinate, and we shall assume that the coordination of private-sector expectations upon the determinate equilibrium is unproblematic.

One case in which this condition fails to be satisfied is when the reaction function makes the interest rate a function solely of exogenous state variables. In this case, equilibrium is indeterminate, for essentially the same reason as in the analysis of Sargent and Wallace [30]. When $i_{t+1}$ is an exogenous process, the endogenous variables $\left\{\pi_{t+1 \mid t}\right\}_{t=t_{0}}^{\infty}$ and $\left\{x_{t+1 \mid t}\right\}_{t=t_{0}}^{\infty}$ are determined solely by a pair of difference equations obtained by taking the expectation of (2.1) and (2.3) conditional upon information in period $t$. This system can be written in vector form as

$$
z_{t+1 \mid t}=M z_{t}+N \tilde{s}_{t}
$$

for $t \geq t_{0}$, where the column vectors $z_{t}$ and $\tilde{s}_{t}$ are defined as

$$
z_{t} \equiv\left[\begin{array}{c}
\pi_{t+1 \mid t} \\
x_{t+1 \mid t}
\end{array}\right], \quad \tilde{s}_{t} \equiv\left[\begin{array}{c}
u_{t+1 \mid t} \\
r_{t+1 \mid t}^{n}-\bar{r} \\
i_{t+1 \mid t}-\bar{r}
\end{array}\right],
$$

the matrix $M$ is defined as

$$
M \equiv\left[\begin{array}{cc}
1 / \beta & -\kappa / \beta \\
-\sigma / \beta & 1+\kappa \sigma / \beta
\end{array}\right],
$$

and the matrix $N$ has elements that do not matter for our argument.

Using standard methods, this system has a unique bounded solution for the process $\left\{z_{t}\right\}_{t=t_{0}}^{\infty}$ if and only if both eigenvalues of the matrix $M$ have modulus greater than one (in which case the solution would be obtained by "solving forward"). The characteristic equation of $M$ is given by

$$
\mu^{2}-\frac{1+\beta+\kappa \sigma}{\beta} \mu+\frac{1}{\beta}=0
$$

${ }^{29}$ We shall not demand the existence of a unique solution to our linear equation system, when even explosive solutions are counted. In general, in a forward-looking model, no policy rule will have that property. The apparent explosive solutions may not correspond to true rational expectations equilibria. One reason is that the conditions for optimality in the private-sector decision problems underlying our structural equations (2.1) and (2.3) include transversality conditions as well as the first-order conditions to which our structural equations correspond. These additional requirements for optimality are necessarily satisfied by any bounded solution, but may not be satisfied by an explosive solution. Furthermore, our structural equations are really only log-linear approximations to the true (nonlinear) equilibrium conditions; bounded solutions to the log-linearized equations approximate solutions to the exact conditions (in the case of small enough disturbances), but explosive solutions may not correspond to any additional solutions to the exact conditions. Finally, determinacy as defined here implies at least local uniqueness of the equilibrium that we consider, which may be considered a reason for greater confidence that the private sector should coordinate its expectations upon the equilibrium than in the case where a very large number of equilibria exist arbitrarily close to one another (the case of indeterminacy). 
which is easily seen to have two real roots satisfying $0<\mu_{1}<1<1 / \beta<\mu_{2}$. Because $\left|\mu_{1}\right|<$ 1 , the condition for determinacy is not satisfied, and instead there is an infinite number of bounded solutions. Since each solution for the forecastable components can be used to construct an equilibrium process for inflation and the output gap using (2.5) and (2.6), we find that equilibrium is indeterminate. ${ }^{30}$

This means that one cannot implement an optimal equilibrium simply by determining how interest rates should evolve in that equilibrium, as a function of the history of exogenous disturbances, and then committing to that functional relation as a rule for setting the interest rate. Such a policy rule would lead to indeterminacy. But there is a further immediate consequence as well. This is that, in this model, any purely forward-looking decision procedure implies a reaction function that results in indeterminacy of equilibrium if the central bank is committed to this procedure. For as argued above, any purely forward-looking procedure implies a reaction function that responds solely to information about the exogenous disturbance processes.

Thus, the desire to obtain a determinate equilibrium is another reason why a desirable policy rule must involve some degree of history-dependence. In particular, we may now furthermore clarify that it must involve some degree of dependence upon lagged endogenous variableswhereas the mere criterion of consistency with an optimal equilibrium might be satisfied by a policy rule that involved dependence solely upon lagged exogenous disturbances (such as a commitment to (2.37) as an instrument rule).

As a simple example of how dependence upon lagged endogenous variables can bring about determinacy, we may consider a Taylor-type rule which prescribes that the interest rate be set each period at the value

$$
i_{t+1}=\bar{r}+g_{\pi} \pi_{t+1 \mid t}+g_{x} x_{t+1 \mid t}
$$

for some coefficients $g_{\pi}, g_{x} \geq 0 .{ }^{31}$ Substituting this rule into (2.3) to eliminate the interest rate,

\footnotetext{
${ }^{30}$ In particular, let $e$ be the right eigenvector of $M$ associated with eigenvalue $\mu_{1}$, and let $\left\{\bar{z}_{t}\right\}_{t=t_{0}}^{\infty}$ be any bounded solution to (2.40). Then consider the alternative process defined by

$$
z_{t}=\bar{z}_{t}+e \delta_{t}, \quad \delta_{t}=\mu_{1} \delta_{t-1}+\xi_{t},
$$

where $\left\{\xi_{t}\right\}_{t=t_{0}}^{\infty}$ is any bounded random variable such that $\xi_{t+1 \mid t}=0$. Then the process $\left\{z_{t}\right\}_{t=t_{0}}^{\infty}$ constructed in this way is another bounded solution to (2.40). Note that this method works no matter what correlation $\xi_{t}$ may have with innovations in "fundamental" disturbances at date $t$, and no matter how large the variability of $\xi_{t}$ may be. Thus there is an infinite set of bounded equilibria; there is an infinite set of additional equilibria arbitarily close to any given equilibrium; and these equilibria include ones in which the target variables fluctuate in response to completely non-fundamental sources of uncertainty ("sunspot equilibria"), as well as an infinite set of equilibria in which they respond solely to "fundamental" uncertainty, but in differing ways. Furthermore, some of the equilibria involve arbitrarily large variability of both inflation and the output gap, and so arbitrarily large values for the expected loss function (2.7). Thus such a policy rule is quite unappealing, if one worries at all about the possibility of one of the less attractive equilibria being the one that results.

${ }^{31}$ Note that if we assume that prices and output are both entirely predetermined, as in Rotemberg and Woodford
} 
we again obtain an equation system of the form (2.40), with the vector $z_{t}$ defined as in (2.41), but in this case the matrix $M$ is given by

$$
M \equiv\left[\begin{array}{cc}
1 / \beta & -\kappa / \beta \\
-\sigma / \beta+\sigma g_{\pi} & 1+\kappa \sigma / \beta+\sigma g_{x}
\end{array}\right] .
$$

One then observes that both roots of the characteristic equation have modulus greater than one, so that equilibrium is determinate, if and only if

$$
g_{\pi}+\frac{1-\beta}{\kappa} g_{x}>1
$$

Thus, a sufficiently strong response to fluctuations in either inflation or the output gap suffices for determinacy. ${ }^{32}$

Note that a reaction function of the form (2.43) must be interpreted as an instrument rule, rather than as an implied reaction function associated with a targeting rule, because it involves dependence on endogenous variables realized only in period $t$. The possibility of such dependence is an advantage of instrument rules, from the point of view of ensuring determinacy. Note that it is not equivalent for the central bank to commit to responding in this way to its own forecast of these variables during its period- $t$ decision cycle, even though all period- $t$ exogenous disturbances are assumed to be observed at that time. This is because a commitment to respond in period $t+1$ to private-sector actions in period $t$ that deviate from the equilibrium expected by the central bank may be useful in ensuring that equilibria other than that one are not equally consistent with private-sector optimization.

However, as we illustrate below, it is not necessary for determinacy that there be feedback from period- $t$ endogenous variables in the setting of $i_{t+1}$; thus reaction functions of the form (2.36) may also imply a determinate equilibrium. ${ }^{33}$ However, our Taylor-type example shows that in the case of an instrument rule, determinacy can be achieved even with a rule that involves no dependence of the instrument upon lagged variables more than one period in the past; in the

[27] and [28], this rule specifies the interest rate as a function of current inflation and output, as in Taylor's original formulation [40]. In the case that these variables are not entirely predetermined, direct dependence upon current inflation and output would not be possible, as these are not yet observed during the bank's period $t$ decision cycle. We might allow dependence upon the bank's estimates of those variables, $\pi_{t+1, t}$ and $x_{t+1, t}$ - which estimates will in fact always be perfectly accurate, because of (2.5) and (2.6) - but such a rule would be dominated by the one proposed in the text, because of the undesirability of unforecastable interest-rate movements. It should be noted that the analysis of determinacy would proceed in exactly the same way for either version of the rule.

32 Note that the coefficients called for by Taylor [40], namely $g_{\pi}=1.5$ and $g_{x}=0.5$, necessarily imply determinacy. More generally, such a rule results in determinacy if and only if it respects what Woodford [47, chapter 2], calls the "Taylor principle": the requirement that a sustained increase in the rate of inflation must eventually result in an increase in the nominal interest rate of an even greater size. Since (2.1) implies that a unit permanent increase in inflation implies a permanent increase in the output gap of $(1-\beta) / \kappa$ units, a rule of the form (2.43) satisfies this principle if and only if (2.45) holds.

${ }^{33}$ See the analysis in section 4 of determinacy in the case of a reaction function of the form (2.38). 
case of a targeting rule, determinacy requires that the reaction function (and hence, the central bank's targets themselves) depend on endogenous variables in period $t-1$ or earlier. Thus, there is a sense in which the required degree of history-dependence is even greater in the case of a targeting rule.

We turn now to an analysis of the consequences of particular decision procedures for monetary policy. We pay particular attention to "forecast targeting" rules, given the reasons for interest in this class of procedures noted in section 1.

\section{Commitment to a modified loss function}

In this section, we discuss our highest-level policy specification, a general targeting rule, which is in terms of a loss function that the central bank is committed to seeking to minimize, through a forecast-based dynamic optimization procedure. We first specify how the central bank computes its forecasts and show the outcome for the optimal forecasts if the central bank uses the social loss function to evaluate these. We show that selecting the optimal forecasts under complete discretion results in a time-consistency problem. One way to restore time-consistency is to apply dynamic programming, and resort to forecasts consistent with the inefficient equilibrium resulting from discretionary optimization (as characterized, for example, using the method of Söderlind [32]). A more attractive way to restore time-consistency is a general targeting rule in the form of a modified loss function, the minimization of which results in forecasts consistent with the optimal equilibrium. We then discuss issues connected with implementation of the optimal equilibrium under this approach.

\subsection{Forecast targeting}

All of the procedures that we discuss in this section involve a particular approach to dynamic optimization, that we call "forecast targeting". Under forecast targeting, the central bank first constructs conditional inflation, output-gap, and interest-rate forecasts corresponding to alternative feasible policies, and then chooses the preferred scenario according to the specified loss function. (A similar procedure is used in the case of our discussion in the next section of specific targeting rules, except that the preferred scenario is chosen as the one that satisfies a specified target criterion.) Let $i^{t} \equiv\left\{i_{t+\tau, t}\right\}_{\tau=1}^{\infty}$ denote such an interest-rate path considered in period $t$, where $i_{t+\tau, t}$ denotes the interest rate considered for period $t+\tau, \tau \geq 1$. Let $\pi^{t} \equiv\left\{\pi_{t+\tau, t}\right\}_{\tau=1}^{\infty}$ and $x^{t} \equiv\left\{x_{t+\tau, t}\right\}_{\tau=1}^{\infty}$ denote conditional (mean) inflation and output-gap 
forecasts (forecast paths) considered in period $t$. We use the notation $\pi_{t+\tau, t}$ and $x_{t+\tau, t}$ to distinguish the central bank's internal forecast in period $t$ for period $t+\tau$ from private-sector inflation and output-gap expectations in period $t$ for period $t+\tau, \pi_{t+\tau \mid t}$ and $x_{t+\tau \mid t}$.

The forecast paths in period $t$ will be related according to the central bank's forecast model,

$$
\begin{aligned}
\pi_{t+\tau, t} & =\beta \pi_{t+\tau+1, t}+\kappa x_{t+\tau, t}+u_{t+\tau, t}, \\
x_{t+\tau, t} & =x_{t+\tau+1, t}-\sigma\left(i_{t+\tau, t}-\pi_{t+\tau+1, t}-r_{t+\tau, t}^{n}\right)
\end{aligned}
$$

for $\tau \geq 1$. Here $u^{t} \equiv\left\{u_{t+\tau, t}\right\}_{\tau=1}^{\infty}$ and $r^{n t} \equiv\left\{r_{t+\tau, t}^{n}\right\}_{\tau=1}^{\infty}$ denote the central bank's (mean) forecasts of the exogenous shocks to the aggregate-supply equation and the natural interest rate, conditional on information available in period $t$ (that is, $u_{t+\tau, t} \equiv \mathrm{E}_{t} u_{t+\tau}$ and $r_{t+\tau, t}^{n} \equiv \mathrm{E}_{t} r_{t+\tau}^{n}$ for $\tau \geq 1$ ). The set of paths satisfying these conditions are the ones over which the bank then optimizes. $^{34}$

\subsection{Discretionary minimization of the social loss function}

Let us first examine the situation when the central bank uses the social loss function to evaluate alternative forecast paths, and chooses as its preferred forecast the one that minimizes the corresponding expected loss. In this case, the central bank's period loss function over the conditional forecasts can be written

$$
L_{t+\tau, t}=\frac{1}{2}\left[\pi_{t+\tau, t}^{2}+\lambda\left(x_{t+\tau, t}-x^{*}\right)^{2}\right]
$$

for $\tau \geq 1$, where in equilibrium $L_{t+\tau, t}$ will differ from $\mathrm{E}_{t} L_{t+\tau}$ by a constant. Thus, in period $t$ the central bank wishes to find the combination $\left(i^{t}, \pi^{t}, x^{t}\right)$ of an interest-rate path and conditional forecasts that fulfills (3.1) and (3.2) and minimizes

$$
L_{t}+\sum_{\tau=1}^{\infty} \beta^{\tau} L_{t+\tau, t},
$$

where $L_{t}$, given by $(2.8)$, is predetermined.

Note that once the central bank has determined its forecasts of the cost-push shock and the natural interest rate, $u^{t}$ and $r^{n t}$, this is a deterministic optimization problem, in contrast

\footnotetext{
${ }^{34}$ Constructing conditional forecasts in a backward-looking model (that is, a model without forward-looking variables) is straightforward. Constructing such forecasts in a forward-looking model raises some specific difficulties, discussed in Svensson [36, appendix A]. The conditional forecasts for an arbitrary interest-rate path derived in the present paper and in Svensson [36, appendix A] assume that the interest-rate paths are "credible", that is, anticipated and allowed to influence the forward-looking variables. A different approach to constructing conditional inflation forecasts for arbitrary interest-rate paths is used by Leeper and Zha [19], who assume that these interest-rate paths result from unanticipated deviations from a normal reaction function.
} 
to the stochastic optimization problem examined above in section 2.1. Furthermore, for any conditional forecasts $\pi^{t}$ and $x^{t}$, the corresponding interest-rate path $i^{t}$ can be constructed from (3.2) by solving for $i_{t+\tau, t}$,

$$
i_{t+\tau, t}=r_{t+\tau, t}^{n}+\pi_{t+\tau+1, t}+\frac{1}{\sigma}\left(x_{t+\tau+1, t}-x_{t+\tau, t}\right) .
$$

Therefore, the central bank can solve the problem in two steps. First, it considers $x_{t+\tau, t}$ as a control variable, and chooses it so that $x^{t}$ and $\pi^{t}$ fulfill (3.1) and minimize (3.4). Second, it calculates the corresponding $i^{t}$ according to (3.5).

The first step can be executed by formulating the Lagrangian

$$
\mathcal{L}_{t} \equiv \sum_{\tau=1}^{\infty} \beta^{\tau}\left\{\begin{array}{c}
\frac{1}{2}\left[\pi_{t+\tau, t}^{2}+\lambda\left(x_{t+\tau, t}-x^{*}\right)^{2}\right] \\
+\Xi_{t+\tau, t}\left[\beta \pi_{t+1+\tau, t}+\kappa x_{t+\tau, t}+u_{t+\tau, t}-\pi_{t+\tau, t}\right]
\end{array}\right\}
$$

where $\Xi_{t+\tau, t}$ is the Lagrange multiplier for the constraint (3.1) for period $t+\tau$, considered in period $t$. Differentiating with respect to $\pi_{t+\tau, t}$ and $x_{t+\tau, t}$ gives the first-order conditions

$$
\begin{aligned}
\pi_{t+\tau, t}-\Xi_{t+\tau, t}+\Xi_{t+\tau-1, t} & =0 \\
\lambda\left(x_{t+\tau, t}-x^{*}\right)+\kappa \Xi_{t+\tau, t} & =0
\end{aligned}
$$

for $\tau \geq 1$, together with the initial condition

$$
\Xi_{t, t}=0 \text {. }
$$

Eliminating the Lagrange multipliers in (3.7) and (3.8) leads to the consolidated first-order condition

$$
\pi_{t+\tau, t}+\frac{\lambda}{\kappa}\left(x_{t+\tau, t}-x_{t+\tau-1, t}\right)=0
$$

for $\tau \geq 2$ and

$$
\pi_{t+1, t}+\frac{\lambda}{\kappa}\left(x_{t+1, t}-x^{*}\right)=0
$$

for $\tau=1$. Thus, finding the optimal forecasts reduces to the problem of finding $\pi^{t}$ and $x^{t}$ that satisfy (3.1), (3.10) and (3.11).

As noted in Woodford [45], these first-order conditions define a decision procedure that will not be time-consistent. This can be seen from the fact that the first-order condition for $\tau=1$, (3.11), is different from that for $\tau \geq 2$, (3.10). This results because, in deciding on $\pi_{t+1, t}$, the central bank takes the previous period's forecast $\pi_{t+1, t-1}$ as given, and lets $\pi_{t+1, t}$ deviate from it without assigning any specific cost to doing so. As a result, the forecasts in period $t$ are 
not generally consistent with the forecasts made in period $t-1$, even if no new information is received in period $t$.

To see this, suppose that the forecasts $\pi^{t-1}$ and $x^{t-1}$ were constructed in period $t-1$ so as to minimize the intertemporal loss function (3.4) with $t-1$ substituted for $t$. The same procedure in period $t-1$ as above then resulted in the same first-order conditions (3.10) and (3.11), although with $t-1$ substituted for $t$. Thus, in period $t-1$, the first-order condition for $\tau=2$ was

$$
\pi_{t+1, t-1}+\frac{\lambda}{\kappa}\left(x_{t+1, t-1}-x_{t, t-1}\right)=0 .
$$

Without any new information in period $t$ relative to period $t-1$, we should have $\pi_{t+1, t}=\pi_{t+1, t-1}$ and $x_{t+1, t}=x_{t+1, t-1}$ for intertemporal consistency. From (3.11) and (3.12) it is apparent that this will not be the case, unless by chance $x_{t, t-1}=x^{*}$.

This illustrates that the period- $t$ forecasts for period- $t+1$ inflation under the above procedure will generally differ from the forecasts of period- $t+1$ inflation in period $t-1$. This also implies that when there is reoptimization in period $t+1$, with new optimal forecasts constructed then, the period- $t+1$ forecast of period- $t+2$ inflation, $\pi_{t+2, t+1}$, would normally differ from the period- $t$ forecast. Thus, the above procedure will not result in time-consistent forecasts, and will violate the intuitive condition stated in Svensson [35], according to which "if no new information has arrived, the forecasts and the interest rate path [should be] the same, and interest setting [should follow] the same interest rate path."

\subsection{A dynamic-programming procedure}

One way to make the forecasts time-consistent would be for the central bank to recognize in period $t$ that the forecasts will be reoptimized in period $t+1$, and to incorporate this in its forecasts in period $t$. This would amount to application of the dynamic-programming approach assumed in standard expositions of the Markov equilibrium resulting from discretionary optimization in a model like ours (such as Söderlind [32]). Under this alternative approach, the first-order conditions (3.7) and (3.8) for the forecasts in period $t$ will instead take the form

$$
\begin{aligned}
\pi_{t+\tau, t}-\Xi_{t+\tau, t} & =0, \\
\lambda\left(x_{t+\tau, t}-x^{*}\right)+\kappa \Xi_{t+\tau, t} & =0,
\end{aligned}
$$

or, equivalently,

$$
\pi_{t+\tau, t}+\frac{\lambda}{\kappa}\left(x_{t+\tau, t}-x^{*}\right)=0
$$


for $\tau \geq 1$.

Using (3.15) in (3.1) and solving in the usual manner, we find in this case that the optimal forecast paths are given by

$$
\begin{aligned}
& x_{t+\tau, t}=\frac{\lambda(1-\beta)}{\kappa^{2}+\lambda(1-\beta)} x^{*}-\frac{\kappa}{\kappa^{2}+\lambda(1-\beta \rho)} \rho^{\tau} u_{t}, \\
& \pi_{t+\tau, t}=\frac{\lambda \kappa}{\kappa^{2}+\lambda(1-\beta)} x^{*}+\frac{\lambda}{\kappa^{2}+\lambda(1-\beta \rho)} \rho^{\tau} u_{t} .
\end{aligned}
$$

One may verify that in this case the forecasts are now intertemporally consistent.

The corresponding instrument path $i^{t}$ is then given by (3.5). It follows that in the period- $t$ decision cycle, the central bank will plan to set the interest rate in period $t+1$ according to

$$
i_{t+1}=i_{t+1, t}=\frac{\lambda \kappa}{\kappa^{2}+\lambda(1-\beta)} x^{*}+\bar{r}+\omega\left(r_{t}^{n}-\bar{r}\right)+\frac{\rho \lambda+(1-\rho) \kappa / \sigma}{\kappa^{2}+\lambda(1-\beta \rho)} \rho u_{t} .
$$

In at least one possible equilibrium associated with this procedure, private-sector plans agree with the forecasts, $\pi_{t+1 \mid t}=\pi_{t+1, t}$ and $x_{t+1 \mid t}=x_{t+1, t}$. In this equilibrium, the forecastable components of inflation and the output gap evolve according to

$$
\begin{aligned}
x_{t+1 \mid t} & =\frac{\lambda(1-\beta)}{\kappa^{2}+\lambda(1-\beta)} x^{*}-\frac{\kappa}{\kappa^{2}+\lambda(1-\beta \rho)} \rho u_{t}, \\
\pi_{t+1 \mid t} & =\frac{\lambda \kappa}{\kappa^{2}+\lambda(1-\beta)} x^{*}+\frac{\lambda}{\kappa^{2}+\lambda(1-\beta \rho)} \rho u_{t} .
\end{aligned}
$$

This equilibrium differs from the optimal equilibrium, described by (2.26) and (2.27), in several respects. First, as long as $x^{*}>0$, there is an average inflation bias, since $\mathrm{E}\left[\pi_{t+1}\right]>0$. Second, the average output gap is positive, $\mathrm{E}\left[x_{t}\right]>0 .{ }^{35}$ Third, the equilibrium lacks historydependence, since $\pi_{t+1 \mid t}$ and $x_{t+1 \mid t}$ do not depend on the past output-gap plan $x_{t \mid t-1}$ or past disturbances $u_{t-j}$. Fourth, the coefficients on $u_{t}$ are different, illustrating the "stabilization bias" discussed in Jonsson [12], Svensson [34], Clarida, Galí and Gertler [4] and Woodford [44].

We shall not examine the actual implementation of such an equilibrium further. Let us just note that (3.16) implies that the interest rate will be a function of the exogenous disturbances. If the private sector perceives of this setup as just being characterized by the reaction function (3.16) and the model equations (2.1) and (2.3), then it follows from the argument of section 2.4 that equilibrium is indeterminate. Suppose instead that the private sector forms expectations in

\footnotetext{
${ }^{35}$ The aggregate-supply equation (2.1) has the property that the long-run Phillips curve is positively sloped, $\mathrm{E}\left[\pi_{t}\right]=\kappa \mathrm{E}\left[x_{t}\right] /(1-\delta)$. This is because the assumption in the standard Calvo setup that firms between optimizing price changes keep their nominal price fixed. If instead, as in Yun [48], it is assumed that prices between optimizing price changes are indexed to the average inflation rate, the long-run Phillips curve is vertical. (Similarly, in the standard Rotemburg setup, it is assumed that any price change is costly, making the long-run Phillips curve positively sloped. If instead it is assumed that any price change different from the average inflation rate is costly, the long-run Phillips curve is vertical.)
} 
accordance with the belief that, in a discretion equilibrium, inflation and the output gap in period $t+2$ should only depend on the exogenous disturbances. Then the private-sector expectations $\pi_{t+2 \mid t}$ and $x_{t+2 \mid t}$ in (2.1) and (2.3) are given exogenously, and private-sector expectations $i_{t+1 \mid t}$ determine the plans $\pi_{t+1 \mid t}$ and $x_{t+1 \mid t}$ uniquely. Then the equilibrium is determinate, and the equilibrium described by (3.17) and (3.18) will result.

\subsection{Sequentially constrained optimization}

We now show that a forecast-based optimization procedure can be rendered consistent with the optimal equilibrium, through a suitable modification of the way in which the central bank evaluates alternative forecast paths. As indicated in our discussion in section 2.1, a suitable procedure must incorporate history-dependence of a kind that is lacking in the procedures discussed in the previous section. One way of introducing the sort of history-dependence that is required is for the central bank to commit itself to internalize the cost of systematically departing from its own previous forecasts. As we have seen in the previous section, the existence of a motive for such deviations is the reason for the suboptimality of a procedure aimed at minimization of the social loss function.

In the case of a deterministic environment, it would be sufficient to add the condition

$$
\pi_{t+1, t}=\pi_{t+1, t-1}
$$

to the bank's decision problem in period $t$. However, this would be inefficient in the more realistic case where there is some new information each period, and hence good reason to let $\pi_{t+1, t}$ deviate from $\pi_{t+1, t-1}$, albeit in an unforecastable way. But we may instead imagine a procedure in which the central bank chooses the forecast path that is optimal subject to a constraint of the form

$$
\pi_{t+1, t}=\bar{\pi}_{t}\left(u_{t}\right)
$$

where the value of $\bar{\pi}_{t}\left(u_{t}\right)$ for each possible realization of the disturbance $u_{t}$ is chosen as part of the bank's period- $t-1$ decision.

It is clear that a dynamic-programming approach of this kind can create the necessary history-dependence, at least in principle. As discussed in section 2.2 above, a timelessly optimal equilibrium involves an expected evolution from any date $t$ onward that is optimal subject to a constraint of the form (2.25). Furthermore, as just discussed, the evaluation of expected losses in any possible equilibrium from date $t$ onward requires only a computation of the associated 
forecast paths. Hence, the choice of $i_{t+1, t}$ that should be made at date $t$ in order to implement the timelessly optimal equilibrium can be made solely on the basis of an evaluation of the alternative forecast paths that are consistent with the constraint (3.19), assuming that in possible state at date $t, \bar{\pi}\left(u_{t}\right)$ takes the same value as in $(2.25)$.

In the case of both of the examples of timelessly optimal equilibria discussed in section 2.2 , the required constraint is of the form

$$
\bar{\pi}_{t}\left(u_{t}\right)=\bar{\pi}_{t, t-1}+\frac{\rho c}{1-\beta \rho c}\left(u_{t}-u_{t, t-1}\right),
$$

where the intercept $\bar{\pi}_{t, t-1}$ depends only on the state of the economy in period $t-1 .^{36}$ Thus we may imagine that the central bank commits itself in period $t-1$ to subject itself in the following decision cycle to a constraint of the form (3.20), where the value of $\bar{\pi}_{t, t-1}$ is chosen in period $t-1$. It is the choice of $\bar{\pi}_{t, t-1}$ on the basis of the economy's state in period $t-1$ that creates the desired history-dependence of subsequent policy.

Because it is only $\bar{\pi}_{t, t-1}$ that must be chosen as part of the bank's period- $t-1$ decision cycle, the choice can be made purely on the basis of a selection among alternative possible forecast paths at that time. (Note that the intercept in (3.20) that is consistent with the timelessly optimal equilibrium is just the forecast value $\pi_{t+1, t-1}$ associated with the constrained-optimal forecast path selected by the central bank in its period- $t-1$ decision cycle.) Furthermore, the bank's choice of the appropriate value for $\bar{\pi}_{t, t-1}$, like its choice of the appropriate value for $i_{t, t-1}$, follows from its desire to bring about the constrained-optimal equilibrium, from among those projected to be possible in its period- $t-1$ decision cycle. If and only if the bank selects the value of $\bar{\pi}_{t, t-1}$ in this way will it expect its own constrained optimization procedure in the following decision cycle to lead it to choose to continue the forecast path selected as constrained-optimal in the current decision cycle.

We thus obtain a sequential forecast-based optimization procedure that is consistent with an equilibrium that is optimal from a timeless perspective. (Either of the two timelessly optimal equilibria discussed in section 2.2 can be shown to be consistent with a procedure of this form, as long as one starts with the appropriate constraint in the first period that the procedure is followed.) However, a possible disadvantage of the procedure, from the point of view of

\footnotetext{
${ }^{36}$ Note that the coefficient on $u_{t}$ is the same in both (2.27) and (2.30). This is not accidental; the coefficient must be the same in the case of any timelessly optimal equilibrium. For in any such equilibrium, the evolution of the economy from date $t$ onward satisfies the system consisting of (2.1), (2.3), (2.11) and (2.12) for some initial condition $\Xi_{t-1}$; alternative equilibria differ only in the way that the initial condition is selected. But the initial condition cannot depend on the realized value of $u_{t}$, nor does the equilibrium response of inflation forecasts to unexpected variation in $u_{t}$ depend on the value assigned to $\Xi_{t-1}$.
} 
communication with the public, is that the determination of which among the feasible forecast paths at a given time are consistent with the constraint (3.19) depends on an evaluation of the current disturbance $u_{t}$, and the extent to which this differs from what was previously expected. This means that the numerical value of this disturbance (that is not meaningful outside the context of the bank's structural model) must be discussed as part of the decision about which among the feasible forecast paths should be selected, and not only in the course of generating the set of feasible forecast paths. Furthermore, the procedure requires the bank to discuss its forecast for this variable, and not simply the forecast paths of the target variables (inflation and the output gap) about which the public cares. The need to explicitly discuss this variable and its consequences for the constraint (3.19), if the public is to be able to verify that the central bank is indeed basing its deliberations upon its putative objective, may be considered a difficulty for practical implementation of the proposal.

\subsection{Minimization of a modified loss function: "Commitment to continuity and pre- dictability"}

A closely related approach, that nonetheless avoids the difficulty just mentioned, is to modify the loss function that the central bank uses to evaluate alternative forecast paths, rather than restricting attention to forecast paths that satisfy a constraint of the form (3.19). It follows from familiar Kuhn-Tucker theory that the constrained optimum of the previous section can alternatively be characterized as the optimum of a loss function that includes an additional term corresponding to the constraint. This dual approach is of particular interest in the present case, because the Lagrange multiplier associated with the constraint (3.19) is independent of the value of $u_{t} .{ }^{37}$ This means that the central bank can choose the value of the Lagrange multiplier that will modify its period- $t$ decision problem as part of its period- $t-1$ decision cycle, and again make this decision solely on the basis of a selection among feasible forecast paths at that time. But in this case, there is no need in period $t$ to adjust the value of the multiplier in response to any surprise that may have occurred in the realization of $u_{t}$.

Suppose that the central bank modifies the period loss function $L_{t+\tau, t}$ for $\tau=1$ by adding the term $\Xi_{t, t-1}\left(\pi_{t+1, t}-\pi_{t+1, t-1}\right)$, hence substituting

$$
\tilde{L}_{t+1, t} \equiv \frac{1}{2}\left[\pi_{t+1, t}^{2}+\lambda\left(x_{t+1, t}-x^{*}\right)^{2}\right]+\Xi_{t, t-1}\left(\pi_{t+1, t}-\pi_{t+1, t-1}\right)
$$

\footnotetext{
37 This follows from the fact that the constraint (3.19) corresponds to the self-consistent constraint (2.25) associated with a timelessly optimal equilibrium.
} 
for $L_{t+1, t}$, where $\Xi_{t, t-1}$ is the corresponding Lagrange multiplier from the decision in period $t-1 .{ }^{38}$ Then the first-order conditions are (3.7) and (3.8) for $\tau \geq 1$, where the initial condition (3.9) for $\tau=1$ is replaced by

$$
\Xi_{t, t}=\Xi_{t, t-1}
$$

Since $\Xi_{t, t-1}$ fulfills (3.8) for $\tau=1$ and $t$ replaced by $t-1$,

$$
\Xi_{t, t-1}=-\frac{\lambda}{\kappa}\left(x_{t, t-1}-x^{*}\right),
$$

the consolidated first-order condition (3.11) for $\tau=1$ becomes

$$
\pi_{t+1, t}+\frac{\lambda}{\kappa}\left(x_{t+1, t}-x_{t, t-1}\right)=0
$$

instead of (3.11). That is, the consolidated first-order condition (3.10) holds for $\tau \geq 1$, and not just for $\tau \geq 2$, with the initial condition

$$
x_{t, t}=x_{t, t-1}
$$

for $\tau=1$. Comparison of these first-order conditions with (2.11) and (2.12) indicates that the optimal forecasts $\pi^{t}$ and $x^{t}$ chosen in period $t$ under this procedure correspond to the optimal equilibrium. Hence, choice of $i_{t+1, t}$ to be consistent with these optimal forecast paths will result in a commitment to an interest rate that is consistent with continuation of the stationary optimal equilibrium.

What is the economic interpretation of the multiplier $\Xi_{t, t-1}$ ? From the Lagrangian (3.6), we see that $\Xi_{t, t-1}$ is the marginal loss in period $t-1$ resulting from an increase in the inflation forecast $\pi_{t+1, t-1}$. Adding the term $\Xi_{t, t-1}\left(\pi_{t+1, t}-\pi_{t+1, t-1}\right)$ to the period- $t$ loss function means that the central bank internalizes this cost when making decisions in period $t$. This is perhaps a somewhat abstract consideration for the purposes of practical policymaking, but it is very much in line with the continuity, predictability and transparency emphasized in actual inflation targeting (see, for instance, King [17]). Hence, we refer to this case as a "commitment to continuity and predictability."

\subsubsection{An explicit decision procedure}

We turn now to an explicit, algorithmic description of the central bank's decision procedure under this proposal. At the beginning of the period- $t$ decision cycle, we suppose that the central

\footnotetext{
${ }^{38}$ Adding a linear term to the loss function is similar to the linear inflation contracts discussed in Walsh [43] and Persson and Tabellini [26]. Indeed, the term added in (3.21) corresponds to a state-contingent linear inflation contract, which, as discussed in Svensson [34], can remedy both stabilization bias and average-inflation bias.
} 
bank observes the current realizations of the exogenous disturbances, which it may use as an input for its decisions; in particular, it observes the values of the current conditional expectations $u^{t}$ and $r^{n t}$. It also recalls its commitment $i_{t, t-1}$, chosen during the previous cycle, and the value assigned to $\Xi_{t, t-1}$.

The first step in the decision procedure is the computation, using the bank's forecasting model, of the set of possible conditional forecasts $\pi^{t}$ and $x^{t}$ that are consistent with the model, given the conditional expectations $u^{t}$ and $r^{n t}$. In our example, these are the paths consistent with (3.1) for all $\tau \geq 1$. It then evaluates the modified loss function, obtained by substituting (3.21) into (3.4), for each possible joint forecast path. In this way, the optimal forecasts are determined as well as the new value of the Lagrange multiplier, $\Xi_{t+1, t}$.

In our example, these optimal forecasts are the ones that satisfy the consolidated first-order condition (3.10) for all $\tau \geq 1$, with the initial condition (3.25). Using (3.10) to eliminate $\pi_{t+\tau, t}$ in (3.1) for $\tau \geq 1$, we get the same second-order difference equation for $x_{t+\tau, t}$ as obtained above for $x_{t+1 \mid t}$, namely (2.16), but with the initial condition (3.25) instead of (2.18). Thus, the characteristic equation again has the two eigenvalues $c$ and $1 /(\beta c)$, where $0<c<1$, and the solution can be written

$$
x_{t+\tau, t}=-\frac{\kappa}{\lambda} c \sum_{j=0}^{\infty}(\beta c)^{j} u_{t+\tau+j, t}+c x_{t+\tau-1, t}
$$

for $\tau \geq 1$.

Since the forecasts $u_{t+\tau, t}$ are given by the true (exogenous) conditional expectations $u_{t+\tau \mid t}$, assumed known to the bank as an input to the process, the term $\sum_{j=0}^{\infty}(\beta c)^{j} u_{t+\tau+j, t}$ has a uniquely determined value. Under the assumption (2.2), this value is simply $\rho^{\tau} /(1-\beta \rho c)$ times the current disturbance $u_{t}$, and (3.26) becomes

$$
\begin{aligned}
x_{t+\tau, t} & =-\frac{\kappa}{\lambda} \frac{c \rho^{\tau}}{1-\beta \rho c} u_{t}+c x_{t+\tau-1, t} \\
& =-\frac{\kappa}{\lambda} \frac{\rho c}{1-\beta \rho c} \frac{c^{\tau}-\rho^{\tau}}{c-\rho} u_{t}+c^{\tau} x_{t, t-1}
\end{aligned}
$$

for each $\tau \geq 1$. From (3.10) it then follows that the optimal forecast of inflation is given by

$$
\begin{aligned}
\pi_{t+\tau, t} & =\frac{\rho c \rho^{\tau}}{1-\beta \rho c} u_{t}+\frac{\lambda}{\kappa}(1-c) x_{t+\tau-1, t} \\
& =\frac{\rho c}{1-\beta \rho c} \frac{(1-\rho) \rho^{\tau-1}-(1-c) c^{\tau-1}}{c-\rho} u_{t}+\frac{\lambda}{\kappa}(1-c) c^{\tau-1} x_{t, t-1}
\end{aligned}
$$

for each $\tau \geq 1$. 
In a third step, the central bank calculates the corresponding forecast path for its instrument, $i^{t}$, according to (3.5). From (3.10), this must satisfy

$$
i_{t+\tau, t}=r_{t+\tau, t}^{n}+\frac{\lambda \sigma-\kappa}{\lambda \sigma} \pi_{t+\tau+1, t}
$$

for $\tau \geq 1$. The forecast path for the natural rate of interest is given by the true conditional expectations (exogenous and known to the bank), while the forecast path for inflation is determined as above. In the case that the disturbance processes satisfy both (2.2) and (2.4), the interest-rate path is given by

$$
i_{t+\tau, t}=\bar{r}+\omega^{\tau}\left(r_{t}^{n}-r\right)+\frac{\lambda \sigma-\kappa}{\lambda \sigma} \frac{\rho c}{1-\beta \rho c} \frac{(1-\rho) \rho^{\tau}-(1-c) c^{\tau}}{c-\rho} u_{t}+f c^{\tau-1} x_{t, t-1}
$$

for each $\tau \geq 1$.

Finally, the central bank makes its decisions. Its action - the setting of its operating target $i_{t}$ for the current period - is determined by the commitment made during the previous decision cycle: it simply sets $i_{t}=i_{t, t-1}$. Its non-trivial current decisions are the selection of a commitment $i_{t+1, t}$ for its action in the following period, and a value for the Lagrange multiplier $\Xi_{t+1, t}$ to be used in the following period's modified loss function. These values are both obtained as initial elements of the forecast paths just computed. Thus, in the case of $\operatorname{AR}(1)$ disturbances the decisions are

$$
\begin{aligned}
i_{t+1, t} & =\bar{r}+\omega\left(r_{t}^{n}-\bar{r}\right)+\frac{\lambda \sigma-\kappa}{\lambda \sigma} \frac{\rho c}{1-\beta \rho c}(c+\rho-1) u_{t}+f x_{t, t-1} \\
\Xi_{t+1, t} & =-\frac{\lambda}{\kappa}\left(x_{t+1, t}-x^{*}\right) \\
& =\frac{\lambda}{\kappa} x^{*}+\frac{\rho c}{1-\beta \rho c} u_{t}-\frac{\lambda}{\kappa} c x_{t, t-1},
\end{aligned}
$$

where we have used (3.8) and (3.28) for $\tau=1$. These decisions are recorded for use as inputs in the following decision cycle. At the beginning of period $t+1$, the new realizations of the exogenous disturbances are observed, and the cycle is repeated.

Several comments about this modified forecast-targeting process are appropriate. One is that the forecast paths that are constructed in successive decision cycles are now time-consistent, in the sense that the forecasts made in decision cycle $t$ coincide with the forecast that the bank would make in period $t$ of what it will forecast using this procedure during any later decision cycle. For example, the bank's forecast in period $t$ of the forecast path for inflation $\pi^{t+1}$ during 
the following decision cycle, denoted $\left[\pi_{t+\tau, t+1}\right]_{, t}$, should be

$$
\begin{aligned}
{\left[\pi_{t+\tau, t+1}\right]_{, t}=} & \frac{\rho c}{1-\beta \rho c} \frac{(1-\rho) \rho^{\tau-2}-(1-c) c^{\tau-2}}{c-\rho} u_{t+1, t}+\frac{\lambda}{\kappa}(1-c) c^{\tau-2} x_{t+1, t} \\
= & \frac{\rho c}{1-\beta \rho c} \frac{(1-\rho) \rho^{\tau-2}-(1-c) c^{\tau-2}}{c-\rho} \rho u_{t} \\
& \quad-(1-c) c^{\tau-2}\left[\frac{\rho c}{1-\beta \rho c} u_{t}+\frac{\lambda}{\kappa} c x_{t, t-1}\right] \\
= & \frac{\rho c}{1-\beta \rho c} \frac{(1-\rho) \rho^{\tau-1}-(1-c) c^{\tau-1}}{c-\rho} u_{t}+\frac{\lambda}{\kappa}(1-c) c^{\tau-1} x_{t, t-1},
\end{aligned}
$$

for each $\tau \geq 2$. Here we have used (3.30) to substitute for $\pi_{t+\tau, t+1}$ in the first line and (3.28) to substitute for $x_{t+1, t}$ in the second. Note that the final line agrees exactly with (3.30), so that the forecasting procedure is consistent.

Furthermore, the bank's forecasts are also consistent with at least one possible equilibrium associated with this policy. The forecasts are, by construction, consistent with (3.1) and (3.2), which are conditions that the true conditional expectations must satisfy in a rationalexpectations equilibrium. In fact one can show that there exists an equilibrium, consistent with the bank's pattern of action under this procedure, in which the true conditional expectations coincide at all times with the bank's forecasts $\left(\pi_{t+\tau \mid t}=\pi_{t+\tau, t}\right.$, and so on). Checking this amounts simply to verifying that the processes

$$
\begin{aligned}
\pi_{t+1} & =\pi_{t+1, t}+u_{t+1}-u_{t+1 \mid t}, \\
x_{t+1} & =x_{t+1, t}+\sigma\left(r_{t+1}^{n}-r_{t+1 \mid t}^{n}\right), \\
i_{t+1} & =i_{t+1, t}
\end{aligned}
$$

satisfy (2.1) and (2.3), when the bank forecasts are constructed as described above.

The equilibrium with this property is also observed to be one that is optimal from the point of view of the timeless perspective defined in section 2.2. Specifically, if the policy regime begins in some period $t_{0}$, with initial conditions $\Xi_{t_{0}, t_{0}-1}$ and $i_{t_{0}, t_{0}-1}$ consistent with the stationary optimal equilibrium, and is expected to continue forever, the equilibrium just described for periods $t \geq t_{0}$ corresponds to the continuation of the stationary optimal equilibrium. The hypothesized initial conditions are, by (3.8) and (2.26),

$$
\begin{aligned}
\Xi_{t_{0}, t_{0}-1} & =-\frac{\lambda}{\kappa}\left(x_{t_{0} \mid t_{0}-1}-x^{*}\right) \\
& =\frac{\lambda}{\kappa} x^{*}+\frac{\rho c}{1-\beta \rho c} \sum_{j=0}^{\infty} c^{j} u_{t_{0}-j-1}
\end{aligned}
$$


and $i_{t_{0}, t_{0}-1}=i_{\dot{t}_{0}}^{*}$. Substitution of these initial conditions into the equations just derived is easily seen to result in exactly the stationary optimal equilibrium characterized in section 2.2. Furthermore, regardless of the initial conditions, the equilibrium involves the optimal responses to shocks that occur from period $t_{0}$ onward, as well as the optimal long-run average values for the endogenous variables. ${ }^{39}$

Note that this procedure need not require that the bank's decisions regarding $i_{t+1, t}$ and $\Xi_{t+1, t}$ be made public, nor that it announce any other aspects of the forecast paths that it constructs as part of the above decision procedure. It need simply set its instrument in the way that has been specified, and, if its decision procedure (or rather, the consequences of the procedure) are correctly understood by the private sector, the optimal equilibrium becomes a rationalexpectations equilibrium consistent with this policy. This is because under this procedure the central bank's forecasts (and actions) are a perfectly predictable function of the history of exogenous disturbances, which are already assumed to be observed by the private sector. Thus, revealing the forecasts, or the commitments chosen by the bank on the basis of them, reveals no additional information. ${ }^{40}$

Nonetheless, announcement of the bank's decisions regarding $i_{t+1, t}$ and $\Xi_{t+1, t}$ may be useful in practice. First of all, the bank's commitment to condition its future decisions upon these past findings may be more reliably fulfilled when the commitments have been made public. (Our analysis in the previous paragraph of the irrelevance of the information provided by the announcements treats the bank's commitment to the decision procedure as unproblematic.) And second, the ability of the private sector to accurately forecast future policy (upon which the above calculation of optimal policy relies) may be facilitated by such announcements of the bank's intentions with regard to future decision cycles. (Our analysis in the previous paragraph similarly takes the private sector's correct understanding of the bank's decision procedure as given.) Similar considerations apply with regard to publication of the bank's forecasts. The fact that past forecasts have been made public may strengthen the bank's commitment to minimize the modified loss function rather than the true social loss function; for unconstrained discretionary

\footnotetext{
${ }^{39}$ Note that modification of the loss function to include the additional term in $(3.21)$, in line with the inflation contracts referred to in footnote 38 , suffices to eliminate the average inflation bias resulting from discretionary minimization of the true social loss function, even when the central bank's loss function includes an output gap target $x^{*}>0$. Thus there is no need to also modify the loss function in the way proposed by King [16], setting $x^{*}=0$ even if that is not its true social value. It is thus an appealing feature of this approach that a single modification of the purely discretionary procedure cures both the problems of "average inflation bias" and "stabilization" bias.

40 The bank's forecasts are predictable not simply given the relations between variables that should exist in equilibrium, but regardless of the equilibrium that happens to be realized. For the procedure described above takes as inputs no observations of external reality other than the evolution of the exogenous disturbances, and involves no internal randomization either.
} 
optimization will result in outcomes that systematically disconfirm previous forecasts. And obviously publication of the bank's forecasts makes it easier for the private sector to coordinate its own forecasts with those of the bank, and hence to act in the way assumed by the bank's analysis. $^{41}$

\subsubsection{The implied reaction function and determinacy}

We turn now to the question of whether the optimal equilibrium just discussed is necessarily the one that results from a commitment to the above procedure. In order to analyze this question, it suffices to consider the implied reaction function of this policy rule, that is, the implied mapping from exogenous and predetermined variables (the information of the central bank at the beginning of each decision cycle) to the bank's setting of its instrument. In the example explicitly treated above, the reaction function of the policy rule is given by $i_{t+1}=i_{t+1, t}$ where $i_{t+1, t}$ is given by equation (3.33) and (3.34). Furthermore, by solving (3.28) for $\tau=1$ backwards, we get

$$
x_{t, t-1}=-\frac{\kappa}{\lambda} \frac{\rho c}{1-\beta \rho c} \sum_{j=0}^{\infty} c^{j} u_{t-j-1} .
$$

Combining this with (3.33) makes it obvious that the implied reaction function is given by $i_{t+1}=i_{t+1}^{*}$, where $i_{t+1}^{*}$ is defined above in (2.32). Thus, as discussed in section 2.4, this decision procedure results in indeterminacy.

Thus, while the optimal equilibrium is one possible equilibrium consistent with a commitment to this policy, it is only one of a very large set of possible equilibria, even if we restrict our attention to stationary equilibria. The others are not optimal, involving sub-optimal responses to disturbances (simply due to self-fulfilling expectations), or fluctuations in response to irrelevant "sunspot" variables, or both. Thus, the use of the modified loss function solves one of the problems associated with discretionary minimization of the true social loss function-the procedure is now consistent with the optimal equilibrium - but it does not eliminate the problem of indeterminacy of equilibrium.

Arguably, the likelihood of the economy's settling upon an inefficient equilibrium might be reduced by making public the complete forecast paths calculated by the central bank. In this case the coordination of private-sector expectations upon exactly those announced by the central bank might be a natural "focal point" for the coordination game faced by private-sector agents

\footnotetext{
${ }^{41}$ The central bank has no incentive to announce a different value for $\Xi_{t+1, t}$ in order to manipulate the outcome of subsequent decision cycles. Because doing so would affect private-sector expecations in period $t$ of its future decisions, this would lead to a worse equilibrium from the point of view of period $t$.
} 
deciding which outcome to expect. Nonetheless, this would be only one among a very large set of other possible equilibria of that "game". An alternative policy rule that is equally consistent with the optimal equilibrium, and that makes it the unique (or at least the unique non-explosive) equilibrium is superior (in at least this respect) to a rule that can only make that equilibrium a "natural focal point" among a large set of possible equilibria.

\subsection{A hybrid rule that ensures determinacy}

Determinacy can, however, be ensured in a more reliable way-by committing the bank to a policy that, if correctly understood by the private sector, excludes other equilibria - if the pure targeting procedure described above is modified in a way that introduces some elements of commitment to an instrument rule.

Note that a targeting procedure, as defined above, makes the bank's actions dependent solely upon its own internal forecasts of what will happen as a result of alternative decisions on its part. Such a purely forecast-based procedure implies that the bank takes no note of whether realized inflation and output gaps deviate from its forecasts (in a systematic way), or alternatively, of whether private-sector plans and expectations deviate from central-bank forecasts. But this is not necessarily reasonable behavior; and indeed, actual inflation-targeting central banks do seem to monitor private-sector plans and expectations, as is apparent from their published Inflation Reports.

When private-sector plans and expectations and the realized equilibrium deviate systematically from the central bank's forecasts, one might well suppose that a forecast-targeting central bank should react to this, by letting its interest-rate deviate from what it would otherwise have set. For example, a bank might commit itself not to set $i_{t+1}=i_{t+1, t}$ regardless of whether its forecasts turn out in the meantime to be confirmed, but instead to set the interest rate according to a rule of the form

$$
i_{t+1}=i_{t+1, t}+g_{\pi}\left(\pi_{t+1 \mid t}-\pi_{t+1, t}\right)+g_{x}\left(x_{t+1 \mid t}-x_{t+1, t}\right)
$$

Here $i_{t+1, t}$ no longer represents a commitment made during the period- $t$ decision cycle as to the value of $i_{t+1}$ that will necessarily be set; but it is still the bank's forecast during that decision cycle as to the value that will be set, assuming that the economy continues to evolve in accordance with the bank's predictions.

Rule (3.39) no longer describes a pure targeting rule, in that the bank's instrument setting 
$i_{t+1}$ no longer follows from a pure calculation of what the effects of one choice or another upon the target variables should be. Instead, it has an element of commitment to an instrument rule - an approach under which the central bank adjusts its instrument in a way that it has committed itself to in advance, not because it judges at the time that this action will have a desirable effect, but because it has judged at an earlier time that it would be desirable for the private sector to anticipate behavior of this kind. Nonetheless, this is not a pure instrument rule either (an approach considered further in section 5), as the rule for setting the interest rate involves a time-varying coefficient $i_{t+1, t}$, which is chosen by the central bank through a targeting procedure. It thus represents a sort of hybrid decision procedure.

The values of $i_{t+1, t}, \pi_{t+1, t}$ and $x_{t+1, t}$ in this equation are each chosen by the central bank during its period- $t$ decision cycle. They are all determined through exactly the same forecasting exercise as has been described above. For in forming its forecasts, the bank expects its forecasts to be correct; thus in computing what it expects the consequences of a given choice of $i_{t+1, t}$ to be, it still expects $i_{t+1}$ to equal $i_{t+1, t}$ in equilibrium. Furthermore, this rule is consistent with continuation of the stationary optimal equilibrium, for the same reason that the specific targeting rule described above is; for in the case that equilibrium occurs (as forecast by the central bank), the actions prescribed by (3.39) are identical to those prescribed by the general targeting rule.

However, the two procedures do not prescribe identical behavior out of equilibrium, and they may differ as to the determinacy of equilibrium. When the central bank follows the explicit decision procedure outlined in section 3.5.1, which results in the implied reaction function $i_{t+1, t}=$ $i_{t+1}^{*},(3.39)$ would correspond to

$$
i_{t+1}=i_{t+1}^{*}+g_{\pi}\left(\pi_{t+1 \mid t}-\pi_{t+1, t}\right)+g_{x}\left(x_{t+1 \mid t}-x_{t+1, t}\right) .
$$

This reaction function is such that the central bank first decides on the interest rate plan, $i^{t}=\left\{i_{t+\tau, t}\right\}_{\tau=1}^{\infty}$ consistent with achieving the optimal inflation and output-gap forecasts, $\pi^{t}=$ $\left\{\pi_{t+\tau, t}\right\}_{\tau=1}^{\infty}$ and $x^{t}=\left\{x_{t+\tau, t}\right\}_{\tau=1}^{\infty}$, that minimize the intertemporal loss function modified according to (3.21), which results in $i_{t+1, t}=i_{t+1}^{*}$, as we have seen. If it, after having announced this interest-rate plan, it observes that private-sector plans for inflation and the output gap, $\pi_{t+1 \mid t}$ and $x_{t+1 \mid t}$, deviate from its forecasts, $\pi_{t+1, t}$ and $x_{t+1, t}$, it makes a further adjustment of the interest rate implemented in period $t+1$ according to (3.40). (Note that this is still a reaction function of the form (2.39), though it no longer satisfies the information restriction assumed in (2.36), as a pure targeting rule would.) 
Let us now consider the determinacy of equilibrium under such a commitment. When (3.40) is combined with the expectation of (2.1) and (2.3), the dynamic system can again be written as in (2.40) with vector $z_{t}$ defined as in $(2.41)$, whereas the vector $\tilde{s}_{t}$ of exogenous variables is now given by

$$
\tilde{s}_{t} \equiv\left[\begin{array}{c}
u_{t+1 \mid t} \\
r_{t+1 \mid t}^{n}-\bar{r} \\
\pi_{t+1, t} \\
x_{t+1, t}
\end{array}\right],
$$

where we exploit that the central bank forecasts $\pi_{t+1, t}$ and $x_{t+1, t}$ depend on the exogenous shocks only. The matrix $M$ is given by (2.44). It follows that a sufficient condition for determinacy is that the coefficients $g_{\pi}$ and $g_{x}$ fulfill (2.45). Since the optimal equilibrium is one possible equilibrium, the unique equilibrium must be the optimal one.

In equilibrium, private-sector plans and central-bank forecasts will be equal, so the term in (3.39) that involves the coefficients $g_{\pi}$ and $g_{x}$ will always be zero. The commitment to deviate from $i_{t+1}^{*}$ in proportion to any deviation of private-sector plans from central bank forecasts is an out-of-equilibrium commitment that will not be noted in the equilibrium. The direction of the deviation is intuitive; if private-sector plans for inflation and/or the output gap exceed the central-bank forecasts, the bank responds with tighter policy - a higher interest rate.

Thus, determinacy is possible in the case of a hybrid rule of this kind, regardless of the values of the model's structural parameters; one simply need to choose any values for $g_{\pi}$ and $g_{x}$ that fulfills (2.45), for instance, Taylor's [40] classic values 1.5 and .5, respectively. This illustrates the fact that a commitment to respond to variables that are predetermined, and hence irrevocable, by the time that the bank responds to them may nonetheless be desirable.

\section{Commitment to a specific targeting rule}

In this section, we introduce our second, intermediate-level policy specification. This is in terms of a specific targeting rule, specifying a criterion that the bank's forecast paths for its target variables must satisfy. This kind of rule specifies a relation involving one or more endogenous variables that cannot be directly observed at the time that policy is chosen, and that instead must be forecasted. Furthermore, in the case of a forward-looking model, even forecasting endogenous variables a short time in the future will in general require solving for the model's equilibrium into the indefinite future; thus a forecast of the entire future paths of the various 
variables is required. A decision procedure of this kind is therefore still organized around the construction of forecast paths conditional upon alternative policies, even if explicit optimization is not undertaken. In the case of such a targeting rule, the history-dependence necessary for determinacy and optimality must be introduced through commitment to a rule that involves lagged endogenous variables as well as forecasts of their future values.

A natural candidate for such a specific targeting rule is the consolidated first-order condition (2.14) for all $t \geq t_{0}$. This condition is not only consistent with the optimality in a timeless perspective, but has the property that, if the central bank could arrange for (2.14) to hold for all $t \geq t_{0}$, this condition would determine a unique bounded solution for periods $t \geq t_{0}$ given by equations (2.29) and (2.30).

However, the central bank cannot directly ensure that such a relation between the paths of its target variables is satisfied. It can, however, adjust its policy so as to produce forecast paths that satisfy this condition. Thus, the targeting rule commits the bank to a policy under which its decisions in period $t$ are chosen so that its forecasts satisfy the condition

$$
\left[\pi_{t+\tau \mid t+\tau-1}+\frac{\lambda}{\kappa}\left(x_{t+\tau \mid t+\tau-1}-x_{t+\tau \mid t+\tau-2}\right)\right]_{, t}=0
$$

for all $\tau \geq 1$. This is a targeting rule involving private-sector plans of one-period-ahead inflation and the output gap. Using the facts that, for $\tau \geq 1,\left[\pi_{t+\tau \mid t+\tau-1}\right]_{, t} \equiv \pi_{t+\tau, t}$ and $\left[x_{t+\tau \mid t+\tau-1}\right]_{, t} \equiv$ $x_{t+\tau, t}$ (under the maintained assumption that the bank does not yet observe current privatesector plans or expectations at the time it makes its current forecast), whereas $\left[x_{t \mid t-1}\right]_{, t} \equiv x_{t \mid t-1}$ (under the assumption that lagged private-sector plans and expectations are observable), this is equivalent to ensuring that the bank's period- $t$ forecast paths satisfy the specific targeting rule

$$
\pi_{t+\tau, t}+\frac{\lambda}{\kappa}\left(x_{t+\tau, t}-x_{t+\tau-1, t}\right)=0
$$

for $\tau \geq 1$, with the convention that

$$
x_{t, t} \equiv x_{t \mid t-1}
$$

Thus, the condition depends upon actually observed past private-sector plans in period $t-1$ for the output gap in period $t, x_{t \mid t-1}$. Note that this differs from the case of a commitment to a modified loss function in section 3.5, cf. (3.25). ${ }^{42}$

In order to find the forecasts $\pi^{t}$ and $x^{t}$ that fulfill this specific targeting rule, the bank combines (4.2) and (4.3) with the aggregate-supply relation (3.1). Using (4.2) to eliminate

\footnotetext{
${ }^{42}$ Leitemo [20] examines the consequences in a forward-looking model of another targeting rule, namely that a constant-interest-rate inflation forecast should equal the inflation target at a specified horizon.
} 
$\pi_{t+\tau, t}$, it gets the same second-order difference equation for $x_{t+\tau, t}$ as obtained above in section 3.5, except that the initial condition is (4.3) rather than (3.25). This implies the same solution (3.28) and (3.30), except that they depend on the previous private-sector output-gap plan $x_{t \mid t-1}$ rather than the previous one-period-ahead central-bank output-gap forecast, $x_{t, t-1}$, that is,

$$
\begin{aligned}
x_{t+\tau, t} & =-\frac{\kappa}{\lambda} \frac{\rho c}{1-\beta \rho c} \frac{c^{\tau}-\rho^{\tau}}{c-\rho} u_{t}+c^{\tau} x_{t \mid t-1} \\
\pi_{t+\tau, t} & =\frac{\rho c}{1-\beta \rho c} \frac{(1-\rho) \rho^{\tau-1}-(1-c) c^{\tau-1}}{c-\rho} u_{t}+\frac{\lambda}{\kappa}(1-c) c^{\tau-1} x_{t \mid t-1} .
\end{aligned}
$$

Using this in (3.31) then results in the implied reaction function

$$
i_{t+1, t}=\bar{\imath}_{t+1}
$$

where $\bar{\imath}_{t+1}$ is defined by (2.33) and (2.34). Thus, the implied reaction function differs from that in section 3.5 , where it was given by $i_{t+1, t}=i_{t+1}^{*}$, where $i_{t+1}^{*}$ is defined by $(2.32)$.

\subsection{Determinacy under the specific targeting rule}

We have already observed that the specific targeting rule (4.2) and the implied reaction function (4.6) is consistent with the equilibrium described by equations (2.30) and (2.29), and thus consistent with continuation of the stationary optimal equilibrium if one starts from initial conditions consistent with that equilibrium. However, it remains to be considered whether the proposed policy commitment requires this outcome, under the assumption that the private sector regards the commitment as fully credible.

When the reaction function defined by (2.33) and (2.38) is combined with the expectations of (2.1) and (2.3), the resulting dynamic system can be written as (2.40), but with the column vectors $z_{t}$ and $\tilde{s}_{t}$ now defined as

$$
z_{t} \equiv\left[\begin{array}{c}
\pi_{t+1 \mid t} \\
x_{t+1 \mid t} \\
x_{t \mid t-1}
\end{array}\right], \quad \tilde{s}_{t} \equiv\left[\begin{array}{c}
u_{t+1 \mid t} \\
r_{t+1 \mid t}^{n}-\bar{r}
\end{array}\right],
$$

and the matrix $M$ given by

$$
M \equiv\left[\begin{array}{ccc}
1 / \beta & -\kappa / \beta & 0 \\
-\sigma / \beta & 1+\kappa \sigma / \beta & \sigma f \\
0 & 1 & 0
\end{array}\right]
$$


The eigenvalues are given by the roots of the characteristic equation, which can be written

$$
\mu\left(\mu^{2}-\frac{1+\beta+\kappa \sigma}{\beta} \mu+\frac{1}{\beta}\right)-\sigma f\left(\mu-\frac{1}{\beta}\right)=0 .
$$

For $f=0$, we have the same roots $\mu_{1}$ and $\mu_{2}$ as in the case of an exogenous process for the interest rate (see section 2.4 above), and a third root $\mu_{3}=0$. Hence, by continuity, for small $f$ we again have indeterminacy, since we don't have exactly two roots of modulus above unity. It can be shown that an interval of positive values of $f$ gives determinacy. The necessary and sufficient conditions for determinacy of a dynamic system of this kind are derived in Woodford [47, Prop. C.2, appendix to chapt. 4] and reproduced in appendix A. The interval of determinacy can be written

$$
\min \left(f_{1}, f_{2}\right)<f<\max \left(f_{1}, f_{2}\right)
$$

where $f_{1}$ and $f_{2}$ are the values of $f$ that correspond to equality in conditions (A.1) and (A.2), respectively. They are

$$
f_{1} \equiv \frac{\kappa}{1-\beta} \quad \text { and } \quad f_{2} \equiv \frac{2}{\sigma}+\frac{\kappa}{1+\beta} .
$$

For the case $f_{2}<f_{1}$, the corresponding eigenvalues fulfill $\mu_{3}<-1<0<\mu_{1}<1<1 / \beta<\mu_{2}$.

Comparing (2.34), (4.9) and (4.10), it is clear that determinacy will at best result only in the case of certain (not obviously plausible) parameter values. Once again, a possible interpretation of this result is that it simply means that following the implied reaction function is not by itself sufficient for determinacy. The central bank may need to supply additional information to the private sector in order to facilitate the coordination of private-sector plans and expectations upon the optimal equilibrium. Thus, ensuring determinacy may provide an additional argument for transparency in central-bank decisionmaking.

As discussed above in section 3, it may be useful for the central bank to announce all or part of its forecasts $\pi^{t}, x^{t}$ and $i^{t}$. If these announcements are credible, in the sense that private-sector plans and expectations agree with the announced forecasts, or even expect that others will, the optimal equilibrium will result. Alternatively, the central bank may announce only the targeting rule (4.1) that it intends to follow. If this announcement is credible, in the sense that people expect the bank to succeed in bringing about the target condition, or at least expect others to expect the condition to hold, the optimal equilibrium will again be the only outcome. 


\subsection{A hybrid rule related to the specific targeting rule}

Determinacy can again also be ensured in a more reliable way, by a hybrid rule involving an intuitive out-of-equilbrium commitment. This can be done in a way directly related to the declared specific targeting rule (4.2), so it is still very much in the spirit of a targeting rule.

Consider, the special case of (3.39) in which $g_{\pi}=\frac{\kappa}{\lambda} g_{x}=g>0$. Then the reaction function implied by the hybrid procedure (3.39) and the specific targeting rule takes the form

$$
i_{t+1}=\bar{\imath}_{t+1}+g\left[\pi_{t+1 \mid t}+\frac{\lambda}{\kappa}\left(x_{t+1 \mid t}-x_{t \mid t-1}\right)\right],
$$

where we have used the fact that central-bank forecasts satisfy (4.2) and (4.6) to obtain a reduced-form variant of (3.39). This reaction function is such that the central bank first decides on the interest rate consistent with achieving the specific targeting rule (4.2), corresponding to $i_{t+1, t}=\bar{\imath}_{t+1}$. If it, after having announced this interest-rate plan, it observes that private-sector plans for inflation and the output gap, $\pi_{t+1 \mid t}$ and $x_{t+1 \mid t}$ deviate from the targeting rule (2.14), it makes a further adjustment of the interest rate implemented in period $t+1$, in the proportion $g$ of the deviation from (2.14). (Note that, again, this is still a reaction function of the form (2.39), though it no longer satisfies the information restriction assumed in (2.36), as a pure targeting rule would.)

Let us now consider the determinacy of equilibrium under such a commitment. When (4.11) is combined with the expectation of (2.1) and (2.3), the dynamic system can again be written as in (2.40) with the definition of the vectors $z_{t}$ and $\tilde{s}_{t}$ as in (4.7), but the matrix $M$ is now given by

$$
M \equiv\left[\begin{array}{ccc}
1 / \beta & -\kappa / \beta & 0 \\
-\sigma / \beta+\sigma g & 1+\kappa \sigma / \beta+\lambda \sigma g / \kappa & \sigma f-\lambda \sigma g / \kappa \\
0 & 1 & 0
\end{array}\right] .
$$

The corresponding characteristic equation can be written

$$
\mu\left(\mu^{2}-\frac{1+\beta+\kappa \sigma}{\beta} \mu+\frac{1}{\beta}\right)-\sigma f\left(\mu-\frac{1}{\beta}\right)-\frac{\lambda \sigma}{\kappa} g\left(\mu^{2}-2 a \mu+\frac{1}{\beta}\right)=0,
$$

where we have separated out the terms multiplied by $g$. We recognize that the quadratic equation in the parenthesis multiplied by $g$ is the same as the characteristic equation (2.19) examined above, with roots $c$ and $1 /(\beta c)$ fulfilling $0<c<1<1 / \beta<1 /(\beta c)$. Furthermore, the rest of the characteristic equation is the same as the characteristic equation (4.8) examined above. If $f$ fulfills (4.9), we already have determinacy even if $g \equiv 0$. One can show that, regardless of 
whether $f$ fulfills (4.9) or not, for any given value of $f$, there exists a value $\bar{g}(f)$ such that

$$
g>\bar{g}(f)
$$

is sufficient for determinacy. The value of $\bar{g}(f)$ is given by

$$
\bar{g}(f) \equiv \max \left\{g_{1}(f), g_{2}(f), \min \left[g_{3}(f), g_{4}(f)\right]\right\},
$$

where $g_{1}(f), g_{2}(f), g_{3}(f)$ and $g_{4}(f)$ are the lowest values such that conditions (A.3) holds for $g>g_{1}(f)$, condition (A.4) for $g>g_{2}(f)$, condition (A.5) for $g>g_{3}(f)$ and condition (A.7) for $g>g_{4}(f)$, respectively. In some cases, the critical value is $g_{1}(f) \equiv 1-(1-\beta) f / \kappa$. Preliminary numerical analysis indicate that $\bar{g}(f)$ for most parameters need not be much different from 1 for determinacy.

Since the optimal equilibrium is one possible equilibrium, the unique equilibrium must be the optimal one. In equilibrium, (2.14) will be fulfilled. The commitment to deviate from $\bar{\imath}_{t+1}$ in proportion to any deviation from (2.14) is an out-of-equilibrium commitment that will not be noted in the equilibrium.

Thus, determinacy is possible in the case of a hybrid rule of this kind, regardless of the values of the model's structural parameters; if (4.9) is violated, one simply need to choose any value for $g$ that fulfills (4.14). This illustrates, again, the fact that a commitment to respond to variables that are predetermined, and hence irrevocable, by the time that the bank responds to them may nonetheless be desirable. In section 5, we now turn to a more general discussion of what may be achieved through commitments of this kind.

\subsection{A commitment to a an equivalent specific price-level targeting rule}

As in Svensson [38], the specific targeting rule (4.2) can be expressed as an equivalent price-level targeting rule. Let $p_{t}$ denote (the log of) the price level in period $t$ (so $\pi_{t} \equiv p_{t}-p_{t-1}$ ). First, define a price-level target path in period $t, p^{* t} \equiv\left\{p_{t+\tau, t}^{*}\right\}_{\tau=0}^{\infty}$, according to

$$
\begin{aligned}
p_{t, t}^{*} & \equiv p_{t, t-1}^{*}+p_{t}-p_{t \mid t-1}, \\
p_{t+\tau, t}^{*} & \equiv p_{t, t}^{*} .
\end{aligned}
$$

This price-level target path is conditional on a given one-period-ahead price-level target in period $t-1, p_{t, t-1}^{*}$, to be determined. The target is adjusted by the unanticipated shock to the price level in period $t, p_{t}-p_{t \mid t-1}=u_{t}-u_{t \mid t-1}$, so that some base drift is allowed to occur. 
Second, consider the specific price-level targeting rule for period $t$,

$$
p_{t+1 \mid t}-p_{t+1, t}^{*}+\frac{\lambda}{\kappa} x_{t+1 \mid t}=0 .
$$

By first-differencing (4.18) (hence, assuming that (4.18) holds in period $t-1$ and in all future periods) and using (4.16) and (4.17), we see that (4.18) implies the consolidated first-order condition (2.14). Third, if (4.18) holds for $p_{t, t-1}^{*}$ in period $t-1$, this together with (4.16) and (4.17) implies

$$
p_{t, t}^{*}=p_{t}+\frac{\lambda}{\kappa} x_{t \mid t-1}
$$

Thus if the price-level targeting rule (4.18) is initiated in a period $t_{0}$ and holds for all $t \geq t_{0}$, we can interpret (4.19) as determining the initial starting point $p_{t_{0}, t_{0}}^{*}$ as a function of the predetermined initial price level, $p_{t_{0}}$, and the previous one-period-ahead private-sector outputgap plan, $x_{t_{0} \mid t_{0}-1}$, after which the future price-level target paths are determined by (4.16) and (4.17).

Again, the central bank cannot directly insure that (4.18) is fulfilled, but it can produce forecast paths that fulfill the corresponding specific targeting rule for the price-level and outputgap forecast paths,

$$
p_{t+\tau, t}-p_{t+\tau, t}^{*}+\frac{\lambda}{\kappa} x_{t+\tau, t}=0
$$

for $\tau \geq 1$. That is, the forecast of the price-level gap between the price level and the price-level target should be proportional to the negative of the output-gap forecast.

In order to find the optimal price-level and output-gap forecasts, $p^{t}=\left\{p_{t+\tau, t}\right\}_{\tau=1}^{\infty}$ and $x^{t}$, the central bank combines (4.20) with the aggregate-supply relation (3.1). This leads to the difference equation

$$
\tilde{p}_{t+\tau+2, t}-2 a \tilde{p}_{t+\tau+1, t}+\frac{1}{\beta} \tilde{p}_{t+\tau, t}=-\frac{1}{\beta} u_{t+\tau+1, t}
$$

for $\tau \geq 0$, where

$$
\tilde{p}_{t+\tau, t} \equiv p_{t+\tau, t}-p_{t+\tau, t}^{*}
$$

denotes the price-level-gap forecast, the initial condition is

$$
\begin{aligned}
\tilde{p}_{t, t} & =p_{t}-p_{t, t}^{*} \\
& \equiv p_{t \mid t-1}-p_{t, t-1}^{*},
\end{aligned}
$$


where we have used (4.16), and $a$ is given by (2.17). Under the assumption (2.2), the solution is

$$
\begin{aligned}
\tilde{p}_{t+\tau, t} & =\frac{c \rho^{\tau}}{1-\beta \rho c} u_{t}+c \tilde{p}_{t+\tau-1, t} \\
& =\frac{\rho c}{1-\beta \rho c} \frac{c^{\tau}-\rho^{\tau}}{c-\rho} u_{t}+c^{\tau} \tilde{p}_{t, t}
\end{aligned}
$$

for $\tau \geq 1$. From (4.20), it then follows the output-gap forecast fulfills

$$
\begin{aligned}
x_{t+\tau, t} & =-\frac{\kappa}{\lambda} \frac{c \rho^{\tau}}{1-\beta \rho c} u_{t}-\frac{\kappa}{\lambda} c \tilde{p}_{t+\tau-1, t} \\
& =-\frac{\kappa}{\lambda} \frac{\rho c}{1-\beta \rho c} \frac{c^{\tau}-\rho^{\tau}}{c-\rho} u_{t}-\frac{\kappa}{\lambda} c^{\tau} \tilde{p}_{t, t},
\end{aligned}
$$

and that the inflation forecast is given by

$$
\begin{aligned}
\pi_{t+\tau, t} & =\frac{\rho c \rho^{\tau}}{1-\beta \rho c} u_{t}-(1-c) \tilde{p}_{t+\tau-1, t} \\
& =\frac{\rho c}{1-\beta \rho c} \frac{(1-\rho) \rho^{\tau-1}-(1-c) c^{\tau-1}}{c-\rho} u_{t}-(1-c) c^{\tau-1} \tilde{p}_{t, t} .
\end{aligned}
$$

Using this in (3.2) to find the optimal instrument rate decision in period $t, i_{t+1, t}$, gives

$$
i_{t+1, t}=\tilde{\imath}_{t+1} \equiv \bar{r}+\omega\left(r_{t}^{n}-\bar{r}\right)+\frac{\lambda \sigma-\kappa}{\lambda \sigma} \frac{\rho c}{1-\beta \rho c}(c+\rho-1) u_{t}+\tilde{f} \tilde{p}_{t, t},
$$

where we have assumed (2.4) and where

$$
\tilde{f} \equiv-\frac{\kappa}{\lambda} f \equiv \frac{\kappa-\lambda \sigma}{\lambda \sigma}(1-c) c .
$$

Note that there is a relatively close relation between optimal inflation targeting under commitment and price-level targeting under discretion, previously discussed by Svensson [37], Clarida, Galí and Gertler [4], Svensson and Woodford [39, section 5.2], Vestin [42] and Smets [31].

Note also that (4.21) and (4.22) imply that the instrument responds to the endogenous variable $p_{t \mid t-1}$ and exogenous shocks. This has implications for the determinacy of equilibrium. When the implied reaction function defined by (4.22) is combined with the expectations of (2.1) and (2.3), the resulting dynamic system can be written as (2.40), but with the column vectors $z_{t}$ and $\tilde{s}_{t}$ now defined as

$$
z_{t} \equiv\left[\begin{array}{c}
p_{t+1 \mid t} \\
x_{t+1 \mid t} \\
p_{t \mid t-1}
\end{array}\right], \quad \tilde{s}_{t} \equiv\left[\begin{array}{c}
u_{t+1 \mid t} \\
r_{t+1 \mid t}^{n}-\bar{r} \\
u_{t}-u_{t \mid t-1}
\end{array}\right]
$$

and the matrix $M$ given by

$$
M \equiv\left[\begin{array}{ccc}
1+1 / \beta & -\kappa / \beta & -1 / \beta \\
-\sigma / \beta & 1+\kappa \sigma / \beta & \sigma \tilde{f}+\sigma / \beta \\
1 & 0 & 0
\end{array}\right]
$$


The eigenvalues are given by the roots of the characteristic equation, which can be written

$$
(\mu-1)\left(\mu^{2}-\frac{1+\beta+\kappa \sigma}{\beta} \mu+\frac{1}{\beta}\right)+\frac{\kappa \sigma}{\beta} \tilde{f}=0 .
$$

For $\tilde{f}=0$, we have the same roots $0<\mu_{1}<1<1 / \beta<\mu_{2}$ as in the case of an exogenous process for the interest rate (see section 2.4 above), and a third root $\mu_{3}=1$. One can show that a sufficient condition for determinacy is

$$
0<\tilde{f}<\tilde{f}_{2}
$$

where

$$
\tilde{f}_{2} \equiv 2+\frac{4(1+\beta)}{\kappa \sigma} .
$$

(Conditions (A.3) and (A.5) impliy $\tilde{f}>0$ and $\tilde{f}<\tilde{f}_{2}$, respectively, and condition (A.7) is always fulfilled.) Comparing (4.9), (4.10) and (4.23)-(4.25), we see that the determinacy conditions for the specific price-level targeting rule (4.20) are different from those for the specific (inflation) targeting rule (4.2). But once again, they need not be fulfilled for all reasonable parameter values.

A hybrid price-level targeting rule of the form

$$
i_{t+1}=\tilde{\imath}_{t+1}+g\left(p_{t+1 \mid t}-p_{t+1, t}^{*}+\lambda x_{t+1 \mid t}\right)
$$

can also be considered, with a corresponding condition on $g$ for determinacy.

\section{Commitment to an explicit instrument rule}

As a final possibility, we now consider monetary policy procedures that involve commitment to the achievement of a rule that links the bank's instrument to other variables that are all either exogenous or predetermined at the time that the instrument must be set. Such an explicit instrument rule represents a possible decision procedure that requires no explicit consideration of either forecasts or optimization problems for its implementation. A commitment of this highly specific kind would have the advantage of making private-sector forecasting of future policy, and monitoring of the degree to which the central bank fulfills its commitment, quite straightforward. It also makes it easy to incorporate into the policy rule the sort of history-dependence that is necessary to achieve the optimal equilibrium, and the sort of dependence upon the realized paths of endogenous variables that is necessary in order for equilibrium to be determinate. A rule of this kind with appropriately chosen coefficients may result in a unique non-explosive 
rational-expectations equilibrium in which the responses to all shocks are optimal; indeed, in the absence of restrictions upon the central bank's information set, there will in general be a large multiplicity of instrument rules that are equally desirable in this regard. ${ }^{43}$

Here we are concerned in particular with whether there are explicit instrument rules that lead to a desirable equilibrium and that also have a relatively transparent relation to the central bank's objective. One respect in which this may be true is that the rule may make the instrument a function solely of the paths of target variables. ${ }^{44}$ This is certainly the point of the wellknown proposal of Taylor [40], under which the instrument rate is made a simple function of current measures of inflation and the output gap. However, simply specifying that policy should respond to any and all deviations of target variables from their (constant) target levels does not necessarily make sense, given that in general complete stabilization of all target variables around the target values will not be feasible even in principle. A more sophisticated approach would instead respond to deviations from the particular pattern of fluctuations in the target variables that is optimal.

It is already clear that one type of explicit instrument rule that is definitely not desirable is a commitment to make the nominal interest rate the particular function $i_{t+1}^{*}$ in (2.32) of the history of disturbances that is associated with the "timeless" optimal equilibrium. For a policy rule of this kind makes the nominal interest rate evolve exogenously, with no feedback from the actual realizations of the endogenous variables; and as we have discussed above in section 2.4, any such rule results in indeterminacy. Indeed, commitment to this instrument rule would be equivalent to commitment to the modified dynamic-optimizing procedure described earlier, which as we saw leads to indeterminacy. In the case of a simple commitment to the implied reaction function (2.32), the absence of any possibility of response to private-sector expectations, and of any opportunity for the central bank to persuade the private sector of its own forecasts, is all the clearer. Thus, the equilibrium paths of inflation and output will not be uniquely determined in this case. Rules in the spirit of the Taylor rule, that specify a response to fluctuations in endogenous variables, are clearly preferable from a determinacy point of view (although, if exactly of the form suggested by Taylor [40], they would not be optimal for the economy considered here).

One way of characterizing undesirable fluctuations in the target variables, that has the

\footnotetext{
43 See Woodford [44] for further discussion of this point.

${ }^{44}$ Of course, there is no general reason to expect that an optimal policy rule should involve responses only to information that is revealed by the history of the target variables, as is stressed in Svensson [36] and [38].
} 
advantages of not requiring explicit reference to the particular exogenous shocks that have occurred, and of being robust to alternative assumed shock processes, is to identify them with failures to satisfy the consolidated first-order condition (2.14), the specific targeting rule that characterizes the optimal equilibrium. A commitment to "make the condition hold" each period is not a possible explicit instrument rule; in the bank's period- $t+1$ decision cycle, it is already a matter of fact whether (2.14) has held or not, whereas in its period- $t$ decision cycle, the endogenous variables $\pi_{t+1 \mid t}$ and $x_{t+1 \mid t}$ are not yet observable (as they will depend upon the bank's period- $t$ decision). Nonetheless, the central bank can commit itself to move its instrument in response to whether the first-order condition has been satisfied.

A simple example of such a rule would be

$$
i_{t+1}=\bar{r}+g\left[\pi_{t+1 \mid t}+\frac{\lambda}{\kappa}\left(x_{t+1 \mid t}-x_{t \mid t-1}\right)\right],
$$

where again $g>0$ is a given response coefficient. Such a commitment is similar to a Taylor-type instrument rule, in which the bank responds to the change in the output gap, rather than its current level, as in the characterizations of Fed policy during the Volcker period proposed by Judd and Rudebusch [13] and Orphanides [25]. It is also necessary, of course, to respond to the forecastable components of inflation and the output gap, rather than to the realized values of these variables, in order for the instrument rule to be fully explicit. ${ }^{45}$ Note that this rule is once again one that makes the central bank's action perfectly forecastable one period in advance $\left(i_{t+1}=i_{t+1 \mid t}\right)$, even though there is no advance announcement of the instrument setting (since the central bank does not yet observe $\pi_{t+1 \mid t}$ and $x_{t+1 \mid t}$ during its period- $t$ decision cycle).

What kind of equilibrium would result from credible commitment to such a policy? Taking expectations of (2.1), (2.3), and (5.1) conditional upon public information in period $t$, and eliminating the variable $i_{t+1 \mid t}$, one obtains a system of difference equations that can again be written in the form (2.40), with the definition of the vectors $z_{t}$ and $\tilde{s}_{t}$ as in (4.7) and with the matrix $M$ is now given by

$$
M \equiv\left[\begin{array}{ccc}
1 / \beta & -\kappa / \beta & 0 \\
-\sigma / \beta+\sigma g & 1+\kappa \sigma / \beta+\lambda \sigma g / \kappa & -\lambda \sigma g / \kappa \\
0 & 1 & 0
\end{array}\right]
$$

(again we do not need the details of the matrix $N$ ).

\footnotetext{
45 Taylor's formulation of his proposal is criticized by McCallum [24] on exactly this point. Note that, if we were to assume that both inflation and output are completely predetermined, as in the analysis of Rotemberg and Woodford [27] and [28], rule (5.1) can be expressed in terms of a direct response to the period $t+1$ inflation rate and output gap, like the policy rules analyzed in those papers.
} 
As usual, determinacy requires that $M$ have exactly two eigenvalues with modulus greater than one, corresponding to the two non-predetermined elements of $z_{t}$. Whether this is true depends upon the size of the response coefficient $g$. The matrix $M$ above is equal to that in (4.12) when $f=0$. It follows that the characteristic equation is the same as (4.14) when $f=0$. Thus, the condition for determinacy is $g>\bar{g}(0)$.

It follows that as long as $g>\bar{g}(0)$, there is a unique bounded solution for $z_{t}$, which depends solely upon the predetermined variable $x_{t \mid t-1}$ and expectations in period $t$ regarding the future paths of the exogenous disturbances. In the case that both disturbances are $\operatorname{AR}(1)$ processes, (2.2) and (2.4), this solution is one in which both $\pi_{t+1 \mid t}$ and $x_{t+1 \mid t}$ are linear functions of $x_{t \mid t-1}$, $u_{t}$ and $r_{t}^{n}$. The next question is the extent to which this equilibrium coincides with the optimal one. In fact, we know that it cannot coincide exactly with the optimal one (more precisely: even if we start from initial conditions consistent with the stationary optimal equilibrium, the equilibrium resulting from a commitment to (5.1) will not continue that optimal equilibrium). This is because we have already seen that the stationary optimal equilibrium requires that the term in brackets in (5.1) be zero at all times, while it also requires that $i_{t+1}=i_{t+1}^{*}$ at all times, a quantity that, by (2.32), is generally different from $\bar{r}$.

On the other hand, the determinate equilibrium associated with rule (5.1) may approximate an optimal equilibrium; in particular, one can show that as $g$ is made sufficiently large, the approximation to the optimal equilibrium becomes arbitrarily close. (Specifically, one can show that in the limit as $g \rightarrow+\infty$, this equilibrium approaches the one described by (2.21) and (2.23) for each period, which is to say, the unique equilibrium in which condition (2.14) holds each period.) However, such a policy prescription is unappealing, because of the possibility that small amounts of noise in the bank's measurement of the forecastable components of the goal variables would lead in practice to highly volatile interest rates. ${ }^{46}$

Alternatively, we can make the instrument rule (5.1) consistent with the stationary optimal equilibrium by adding a time-varying intercept term,

$$
i_{t+1}=i_{t+1}^{*}+g\left[\pi_{t+1 \mid t}+\frac{\lambda}{\kappa}\left(x_{t+1 \mid t}-x_{t \mid t-1}\right)\right] .
$$

This is now a rule that is consistent with the stationary optimal equilibrium, regardless of

\footnotetext{
${ }^{46}$ Here we presume that the central bank's measurement error does not become apparent to the private sector, and so cannot affect private sector forecasts or behavior, until after the quantities in the square brackets in (5.1) have been determined. Note that the central bank's error need not become apparent to the private sector until the period $t+1$ interest rate is revealed, whereas the forecasts to which the central bank responds in setting $i_{t+1}$ are all determined by the private sector in period $t$. For further discussion of the undesirability of this approach to stabilization, see Bernanke and Woodford [2].
} 
the value of $g$. Because the added term is an exogenous random process, the determinacy calculations remain the same as above, and we again find that for $g>\bar{g}(0)$, equilibrium is determinate. Since we already know that the optimal equilibrium is consistent with (5.2), it follows from determinacy that the unique bounded equilibrium is an optimal one.

As yet another alternative, we could modify (5.1) by adding an endogenous term that renders the rule consistent with the stationary optimal equilibrium, namely

$$
i_{t+1}=\bar{\imath}_{t+1}+g\left[\pi_{t+1 \mid t}+\frac{\lambda}{\kappa}\left(x_{t+1 \mid t}-x_{t \mid t-1}\right)\right],
$$

where once again $\bar{\imath}_{t+1}$ is defined by (2.33). This is identical to the reaction function (4.11) implied by the hybrid procedure considered above in section 4.2 , though here we contemplate a direct commitment to bring about this reaction function as an explicit instrument rule. The determinacy analysis is the same as in the previous section. Thus, for $g>\bar{g}(f)$, equilibrium is determinate, and the unique bounded equilibrium is an optimal one.

These two examples illustrate the possibility of achieving the optimal equilibrium as a determinate outcome through commitment to an explicit instrument rule with bounded coefficients. They also illustrate an important general point. This is that the mere fact that the target variables are predetermined in the short run, and so not able to be affected by current central bank decisions, does not imply that the only effective procedure must be a forward-looking one, that aims to have a certain effect upon the future paths of the target variables. Instead, as long as the private sector is forward-looking and the central bank's policy rule can be made credible, committing to respond in a purely backward-looking way to past deviations of the target variables from their desired path can be an effective way of reducing the size of those deviations in equilibrium. The anticipation that the central will later respond in this way is enough to achieve the desired effect, and indeed, in a model like that assumed here, it is only the private sector's expectations regarding future policy that can have any effect on the evolution of the target variables at all.

This seems an important principle to keep in mind in choosing a policy rule, especially insofar as the determinacy of equilibrium is a concern. However, the explicit instrument rules proposed above remain unattractive on grounds of robustness. Note that a suitable specification of either the targeting rule (4.1) or the hybrid rule (4.11) depends only upon the slope coefficient $\kappa$ of the aggregate supply relation, and not upon other coefficients of the bank's model of the economy or any details of the assumed specification of the exogenous shock processes. Instead the term $i_{t+1}^{*}$ in (5.2) depends also upon the slope coefficient $\sigma$ of the model's IS relation, and upon the 
parameters of the exogenous shock processes (for instance, in the AR(1) specification assumed in (2.32), upon the parameters $\rho$ and $\omega)$. The same is true of the term $\bar{\imath}_{t+1}$ in (5.3). The presence of these terms also requires that one sacrifice one of the obvious advantages of simple instrument rules like the "Taylor rule", which is ease of communication of the nature of the commitment to the general public. When the instrument rule involves reference to responses to exogenous disturbances (rather than simply to goal variables, that are better understood by the public, and are publicly reported), there is no longer any particular advantage of this approach in terms of transparency.

The hybrid procedure defined by (4.11) is more attractive in both of these last regards. For that specification of the policy commitment depended only upon the specific value of $\kappa$, yet (in the case that the specific model assumed above is used) it implied an identical reaction function as the instrument rule (5.3). It was also a specification that required no explicit reference to the exogenous disturbances. Such a hybrid approach thus combines several of the most attractive features of a specific targeting rule and of an explicit instrument rule.

\section{Concluding remarks}

We now offer a few remarks on the degree to which the various decision procedures discussed above satisfy the desiderata for a desirable monetary policy rule mentioned in the introduction. Our first and most important criterion, of course, is consistency of the policy rule with the stationary optimal equilibrium characterized in section 2. As we have seen, the most naive approach to inflation-forecast targeting - a forecast-based discretionary optimizing procedure aimed at minimization of the true social loss function - fails to have this property. However, we have shown that there are many different ways in which one could introduce the sort of history-dependence required for consistency with the optimal equilibrium. Possible methods include modification of the loss function that the forecast-based optimizing procedure seeks to minimize, commitment to a specific targeting rule such as (4.1), commitment to an instrument rule such as (5.2) or (5.3), or commitment to a hybrid procedure such as (3.39) or (4.11). Any of these approaches would be equally satisfactory from the point of view of consistency with the optimal equilibrium, assuming credibility of the bank's commitment to the rule in question.

Our second criterion was determinacy of equilibrium under the policy rule, so that one could count on the optimal equilibrium being the one which should result from a correct understanding of the central bank's commitment on the part of the private sector. This turned out to be 
a problem for the procedure discussed in section 3.5, directed toward the minimization of a modified loss function, the "commitment to continuity and predictability." In the case of our present model, such a procedure results in indeterminacy for all possible values of the model parameters. And more generally, because such a procedure necessarily corresponds to an implied reaction function involving no dependence upon lagged endogenous variables except insofar as these are relevant to forecasts of the future evolution of the target variables, such rules are less likely to involve the dependence upon lagged endogenous variables that is necessary in order to exclude self-fulfilling expectations.

This problem may be mitigated by a sufficient degree of transparency of the bank's decision procedure, as this may facilitate the coordination of private-sector expectations upon the paths forecasted by the central bank. But this would still seem to be a weakness of our highest-level approach to the specification of a policy rule, relative to lower-level specifications that make the bank's decisions dependent upon lagged endogenous variables for reasons unrelated to their effect upon the bank's forecasts.

However, a way to achieve determinacy is to amend the general targeting procedure with a commitment to a particular instrument-rate response by the central bank, if the privatesector plans of inflation and the output gap deviate from the central bank's forecast. This is the hybrid rule discussed in section 3.6 and represented by equation (3.40). Since this is an out-of-equilibrium commitment, it will not have any observable consequences in equilibrium.

A specific targeting rule can introduce additional dependence upon lagged endogenous variables, through commitment to a target criterion that depends upon past as well as future paths of the target variables. However, in the case of the simple targeting rule (4.1), indeterminacy is likely still to be a problem for reasonable parameter values. Achieving determinacy in this way may require an even greater degree of dependence of the target criterion upon the past history of the target variables. Again, one way to achieve determinacy is to amend the specific targeting rule with a commitment to a particular out-of-equilibrium instrument-rate response by the central bank, if the specific targeting rule is violated. A hybrid rule that serves this purpose has been discussed in section 4.2 and displayed in equation (4.11).

An alternative approach, that can easily result in a determinate equilibrium that is also optimal from our timeless perspective, is commitment to an explicit instrument rule that requires the central bank to respond to deviations of the target variables from a target criterion which they should satisfy in an optimal equilibrium. This is illustrated by the explicit instrument rules 
(5.2) and (5.3), but the hybrid rules mentioned above works equally well in this regard.

However, it should be stressed, that the magnitude of the determinacy problems above may be exaggerated by the extremely forward-looking character of the model assumed here, in which no lagged endogenous variables are relevant to the determination of current and future values of the target variables, except insofar as such dependence is introduced through the monetary policy rule. A consideration of the extent to which the decision procedures of the kind we have considered would still face indeterminacy problems in a more complex, and possibly more realistic, model with sources of intrinsic inertia in the endogenous variables remains a topic for further research.

There remain two further criteria for comparison of our candidate policies. As noted in the introduction, we prefer approaches to monetary policy in which the connection between the central bank's decision process and its ultimate objectives is as transparent as possible. From this point of view, our highest-level policy specifications, in terms of a procedure that aims to minimize a specified loss function, are most suitable. The most transparent procedure would be the naive approach of discretionary minimization of the social loss function; but this procedure, as we have seen, is inconsistent with an optimal equilibrium. Minimizing a modified loss function, the commitment to continuity and credibility discussed in section 3.5, is somewhat less transparent, although the idea of taking into account the shadow cost of the previous centralbank forecasts and private-sector expectations is arguably a direct consequence of the desire to minimize the social loss function, once the nature of the bank's optimization problem is properly understood. Such concerns are also arguably already present in the thinking and rhetoric of actual inflation-targeting central banks, given its emphasis on continuity and predictability (see, for instance, King [17]). However, in a more complex model with a greater number of forwardlooking variables, this approach would imply that the Lagrange multipliers of all of the (relevant) forward-looking variables would need to be recorded and taken into account in modifying the period loss function. This would make the approach far less transparent, and perhaps less practical as well.

The specific targeting rule discussed in section 4 and described by equation (2.14), implying that the expected deviation between inflation and the inflation target should be proportional to the decrease in the predictable component of the output gap, is simple, but somewhat less intuitive, and for that reason less transparently related to underlying policy goals. ${ }^{47}$ The equivalent

\footnotetext{
${ }^{47}$ In at least some very simple models, a similar specific targeting rule derived from the first-order conditions that characterize the optimal equilibrium is more intuitive, and indeed more similar to the sort of intuitive
} 
price-level targeting rule for the forward-looking model discussed in section 4.3 is arguably more intuitive, though. And in any event, because such a rule is still specified in terms of the desired behavior of the target variables, it scores better on this criterion than would instrument rules such as (5.2) and (5.3). The same is true of the hybrid variant of this procedure described by (4.11).

Because explicit instrument rules are formulated as rules of central-bank conduct that happen, generally for relatively indirect reasons, to have desirable consequences if anticipated by the private sector, rather than as descriptions of what the bank is trying to achieve, they rate lowest on the criterion of transparency. A rule such as (5.1), however, is more transparently related to the goals of policy than many other instrument rules would be, insofar as it prescribes response to failure of the target variables to satisfy a target criterion (indeed, the same criterion as is the basis for the specific targeting rule (2.14)). However, as we have seen, (5.1) in its simplest form is not consistent with the optimal equilibrium. Modified instrument rules such as (5.2) and (5.3), that are consistent with optimality, involve fairly complex functions of lagged disturbances or endogenous variables that are clearly not related to the goals of policy in any transparent way.

Our final criterion is the robustness of the alternative monetary-policy procedures to modifications of the assumed model of the economy. The general topic of robustness is beyond the scope of this study, but our results here do allow us to comment upon the sensitivity of the various specifications to changes in parameters while assuming the same basic model structure.

Clearly, the higher-order policy specifications are more robust to model perturbations. Our general approach in section 3.5 of modifying the loss function so as to make a discretionary optimizing procedure consistent with the optimal equilibrium is not dependent upon the details of the bank's model of the economy at all. Only the identification of the relevant forwardlooking variables and their associated Lagrange multipliers is at all model-dependent; nothing about the specification would need to be changed as a result of changes in model parameters that maintained the same basic form of equations (2.1) and (2.3), or changes in the assumed specification of the exogenous disturbance processes.

The specific targeting rule (2.14) is less robust than this, but it still depends only upon the slope coefficient $\kappa$ of the aggregate-supply relation. The targeting rule is independent of the nature and number of the exogenous disturbances in the aggregate-supply equation. And as forecast-targeting rules followed by actual inflation-targeting central banks; see Svensson [33]. 
long as there is no weight on interest-rate stabilization or smoothing in the loss function, the targeting rule is completely independent of both the form of the IS equation and the nature of its disturbances. Thus, the targeting rule arising in this model is quite robust to a number of model perturbations. This supports the conjecture arising in the backward-looking model of Svensson [33] that targeting rules are likely to be more robust than instrument rules. ${ }^{48}$ The hybrid variant of this rule (3.39) is equally robust.

The instrument rules (5.2) and (5.3) are the least robust, since they depend on all of the parameters of the model and are not robust to any perturbations - except changes in the variances of the iid shocks, due to the certainty-equivalence that holds in a linear model with a quadratic loss function.

Overall, we find that each of our general classes of policy specifications contains specifications that incorporate the kind of history-dependence required for consistency with the optimal equilibrium. The lower-level specifications are most advantageous from the point of view of ensuring determinacy, whereas to the contrary, we find that the higher-level specifications are most advantageous from the standpoints of transparency and robustness. An intermediate-level policy specification, involving commitment to a specific targeting rule, may be the best overall compromise among these competing concerns. The hybrid procedure described in section 4.2 is perhaps the most attractive of the alternatives reviewed here, as it allows one to ensure determinacy regardless of the model parameters, while at the same time being quite robust, and retaining a more transparent relation to the goals of policy than is possible in the case of an explicit instrument rule.

\footnotetext{
${ }^{48}$ Svensson [38] takes this argument further, and shows that specific targeting rules are robust to the unavoidable use of judgment in practical monetary policy. Giannoni and Woodford [11] show how robust targeting rules can be computed for a general class of linear-quadratic policy problems.
} 


\section{A. The necessary and sufficient conditions for determinacy}

Consider a system of difference equations of the form,

$$
z_{t+1 \mid t}=M z_{t}+N \tilde{s}_{t}
$$

where $z_{t}$ denotes a vector of three endogenous variables, two of which are forward-looking and one of which is predetermined, $\tilde{s}_{t}$ denotes a vector of exogenous variables, and $M$ and $N$ are matrices of appropriate dimension. The solution to this system is determinate if and only if the matrix $M$ has one eigenvalue with modulus less than one and two eigenvalues with modulus greater than one.

The characteristic equation of the system will be cubic and can be written

$$
\mu^{3}+a_{2} \mu^{2}+a_{1} \mu+a_{0}=0
$$

Woodford [47, Prop. C.2, appendix to chapter 4] shows that the solution to the system is determinate if and only if the coefficients of the characteristic equation fulfill either (case I)

$$
\begin{aligned}
1+a_{2}+a_{1}+a_{0} & <0 \text { and } \\
-1+a_{2}-a_{1}+a_{0} & >0
\end{aligned}
$$

or (case II)and

$$
\begin{aligned}
1+a_{2}+a_{1}+a_{0} & >0, \\
-1+a_{2}-a_{1}+a_{0} & <0 \text { and } \\
a_{0}^{2}-a_{0} a_{2}+a_{1}-1 & >0 ;
\end{aligned}
$$

or (case III) (A.3) and (A.4) hold, together with

$$
\begin{aligned}
a_{0}^{2}-a_{0} a_{2}+a_{1}-1 & <0 \text { and } \\
\left|a_{2}\right| & >3 .
\end{aligned}
$$




\section{References}

[1] Backus, David, and John Driffill (1986), "The Consistency of Optimal Policy in Stochastic Rational Expectations Models," CEPR Discussion Paper No. 124.

[2] Bernanke, Ben, and Michael Woodford (1997), "Inflation Forecasts and Monetary Policy," Journal of Money, Credit, and Banking 24, 653-684.

[3] Clarida, Richard, Jordi Galí and Mark Gertler (1998), "Monetary Policy Rules in Practice: Some International Evidence," European Economic Review 42, 1033-1067.

[4] Clarida, Richard, Jordi Galí and Mark Gertler (1999), "The Science of Monetary Policy: A New Keynesian Perspective," Journal of Economic Literature 37, 1661-1707.

[5] Christiano, Lawrence J., and Christopher J. Gust (1999), "Taylor Rules in a Limited Participation Model," NBER Working Paper No. 7017

[6] Christiano, Lawrence J., and Robert J. Vigfusson (1999), "Maximum Likelihood in the Frequency Domain: A Time to Build Example," NBER Working Paper No. 7027

[7] Currie, David, and Paul Levine (1993), Rules, Reputation and Macroeconomic Policy Coordination, Cambridge University Press, Cambridge.

[8] Edge, Rochelle M. (2000), "Time to Build, Time to Plan, Habit Persistence, and the Liquidity Effect," International Finance Discussion Paper no. 2000-673, Federal Reserve Board, July.

[9] Flodén, Martin (1996), "The Time Inconsistency Problem of Monetary Policy under Alternative Supply Side Modeling," Working Paper, Stockholm University, http://www.hhs.se/personal/floden/.

[10] Giannoni, Marc P. (1999), "Does Model Uncertainty Justify Caution? Robust Optimal Monetary Policy in a Forward-Looking Model," Working Paper, Princeton University.

[11] Giannoni, Marc. P., and Michael Woodford (2002), "Optimal Interest-Rate Rules: I. General Theory," NBER working paper no. 9419, December.

[12] Jonsson, Gunnar (1997), "Monetary Politics and Unemployment Persistence," Journal of Monetary Economics 39, 303-325. 
[13] Judd, John P., and Glenn D. Rudebusch (1998), "Taylor's Rule and the Fed: 1970-1997," FRBSF Economic Review 1998(3), 3-16.

[14] Kerr, William, and Robert J. King (1996), "Limits on Interest Rate Rules in the IS Model," Economic Quarterly, Federal Reserve Bank of Richmond, Spring 1996, 47-76.

[15] King, Mervyn A. (1994), "Monetary Policy in the UK," Fiscal Studies 15, No. 3, 109-128.

[16] King, Mervyn A. (1997), "Changes in UK Monetary Policy: Rules and Discretion in Practice," Journal of Monetary Economics 39, 81-97.

[17] King, Mervyn (1997), "The Inflation Target Five Years On,” Bank of England Quarterly Bulletin 37(4).

[18] Kydland, Finn E., and Edward C. Prescott (1977), "Rules Rather than Discretion: The Inconsistency of Optimal Plans," Journal of Political Economy 85, 473-491.

[19] Leeper, Eric M., and Tao Zha (1999), "Identification and Forecasting: Joint Inputs to Policy Analysis," Working Paper.

[20] Leitemo, Kai (1999), "Targeting Inflation by Constant-Interest-Rate Forecast," Working Paper, Norges Bank.

[21] Levin, Andrew, Wieland, Volker, and John C. Williams (1999), "Robustness of Simple Monetary Policy Rules under Model Uncertainty," in Taylor [41].

[22] McCallum, Bennett T. (1981), "Price Level Determinacy with an Interest Rate Policy Rule and Rational Expectations," Journal of Monetary Economics 8, 319-329.

[23] McCallum, Bennett T. (1999), "Role of the Minimal State Variable Criterion," NBER Working Paper No. 7087.

[24] McCallum, Bennett T. (1997), "Issues in the Design of Monetary Policy Rules," NBER Working Paper No. 6016.

[25] Orphanides, Athanasios (1999), "The Quest for Prosperity without Inflation," Working Paper, Federal Reserve Board.

[26] Persson, Torsten, and Guido Tabellini (1993), "Designing Institutions for Monetary Stability," Carnegie-Rochester Conference Series on Public Policy 39, 53-84. 
[27] Rotemberg, Julio J., and Michael Woodford (1997), "An Optimization-Based Econometric Framework for the Evaluation of Monetary Policy," NBER Macroeconomics Annual 12, $297-346$.

[28] Rotemberg, Julio J., and Michael Woodford (1999), "Interest-Rate Rules in an Estimated Sticky-Price Model," in Taylor [41].

[29] Rudebusch, Glenn D., and Lars E.O. Svensson (1999), "Policy Rules for Inflation Targeting," in Taylor [41].

[30] Sargent, Thomas J., and Neil Wallace (1975), "Rational Expectations, the Optimal Monetary Instrument, and the Optimal Money Supply Rule," Journal of Political Economy 83, $241-254$.

[31] Smets, Frank (2000), "What Horizon for Price Stability," ECB Working Paper No. 24.

[32] Söderlind, Paul (1999), "Solution and Estimation of RE Macromodels with Optimal Policy," European Economic Review 43, 813-823.

[33] Svensson, Lars E.O. (1997a), "Inflation Forecast Targeting: Implementing and Monitoring Inflation Targets," European Economic Review 41, 1111-1146.

[34] Svensson, Lars E.O. (1997b), "Optimal Inflation targets, 'Conservative' Central Banks, and Linear Inflation Contracts," American Economic Review 87, 98-114.

[35] Svensson, Lars E.O. (1999a), "How Should Monetary Policy Be Conducted in an Era of Price Stability?" in New Challenges for Monetary Policy, A Symposium Sponsored by the Federal Reserve Bank of Kansas City, Jackson Hole, Wyoming, August 26-28, 1999.

[36] Svensson, Lars E.O. (1999b), "Inflation Targeting as a Monetary Policy Rule," Journal of Monetary Economics 43, 607-654. Longer working-paper version, including appendix, available at www.princeton.edu/ svensson.

[37] Svensson, Lars E.O. (1999c), "Price-Level Targeting vs. Inflation Targeting: A Free Lunch?" Journal of Money, Credit, and Banking 31, 277-295.

[38] Svensson, Lars E.O. (2003), "What Is Wrong with Taylor Rules? Using Judgment in Monetary Policy through Targeting Rules," Journal of Economic Literature, forthcoming. 
[39] Svensson, Lars E.O., and Michael Woodford (2002), "Indicator Variables for Optimal Policy," Journal of Monetary Economics, forthcoming.

[40] Taylor, John B. (1993), "Discretion versus Policy Rules in Practice," Carnegie-Rochester Conference Series on Public Policy 39, 195-214.

[41] Taylor, John B., ed. (1999b), Monetary Policy Rules, Chicago University Press.

[42] Vestin, David (1999), "Price-Level Targeting versus Inflation Targeting in a ForwardLooking Model," Working Paper, Stockholm University.

[43] Walsh, Carl (1995), "Optimal Contracts for Independent Central Bankers," American Economic Review 85, 150-167.

[44] Woodford, Michael (1999a), "Optimal Monetary Policy Inertia," NBER Working Paper No. 7261, July.

[45] Woodford, Michael (1999b), "Commentary: How Should Monetary Policy Be Conducted in an Era of Price Stability?" in New Challenges for Monetary Policy, A Symposium Sponsored by the Federal Reserve Bank of Kansas City, Jackson Hole, Wyoming, August 26-28, 1999.

[46] Woodford, Michael (2000), "Pitfalls of Forward-Looking Monetary Policy," American Economic Review Papers and Proceedings 90: 100-104.

[47] Woodford, Michael (2003), Interest and Prices: Foundations of a Theory of Monetary Policy, forthcoming, Princeton University Press.

[48] Yun, Tack (1996), "Nominal Price Rigidity, Money Supply Endogeneity, and Business Cycles," Journal of Monetary Economics 37, 345-370. 